\title{
Catalytic Oxidation of Imines Based on Methyltrioxorhenium/Urea Hydrogen Peroxide: A Mild and Easy Chemo- and Regioselective Entry to Nitrones
}

\author{
Gianluca Soldaini, Francesca Cardona and Andrea Goti* \\ Dipartimento di Chimica Organica "Ugo Schiff”, Laboratorio di Progettazione, Sintesi e Studio di \\ Eterocicli Biologicamente Attivi (HeteroBioLab) and Consorzio INSTM, Università di Firenze, \\ Polo Scientifico, via della Lastruccia 13, I-50019 Sesto Fiorentino (Firenze), Italy
}

\section{Experimental}

Commercial reagents were used as received. All reactions were magnetically stirred and monitored by TLC on $0.25 \mathrm{~mm}$ silica gel plates $\left(\mathrm{Merck}_{254}\right)$ and column chromatography was carried out on Silica Gel $60(32-63 \mu \mathrm{m})$. Yields refer to spectroscopically and analytically pure compounds unless otherwise stated. ${ }^{1} \mathrm{H}$ and ${ }^{13} \mathrm{C}$ NMR spectra were recorded on Varian Gemini-200 or Varian Mercury-400. Proton and carbon chemical shifts are reported relative to the residue peaks of the solvent $\mathrm{CDCl}_{3}$ (7.26 ppm for ${ }^{1} \mathrm{H}$ and $77.0 \mathrm{ppm}$ for $\left.{ }^{13} \mathrm{C}\right)$. Infrared spectra were recorded with a Perkin-Elmer Spectrum BX FT-IR System spectrophotometer. Mass spectra were recorded on a QMD 1000 Carlo Erba instrument by direct inlet; relative percentages are shown in brackets. Elemental analyses were performed with a Perkin-Elmer 2400 analyzer. Optical rotation measurements were performed on a JASCO DIP-370 polarimeter.

Imines 2a-r were prepared by condensation of the corresponding aldehydes with amines in $\mathrm{CH}_{2} \mathrm{Cl}_{2}$. Imines $\mathbf{2 s - v}$ were prepared by $\mathrm{HCl}$ elimination from the corresponding chloroamines. ${ }^{1}$

\section{General Procedure for the Oxidation of Imines to Nitrones}

To a stirred solution of the imine $2 \mathbf{a}-\mathbf{v}(1 \mathrm{mmol})$ in $\mathrm{MeOH}(2 \mathrm{~mL})$, UHP $(282 \mathrm{mg}, 3 \mathrm{mmol})$ and MTO (5 mg, $0.02 \mathrm{mmol}$ ) were added sequentially. The resulting yellow solution was stirred at room temperature until disappearance of the starting material (TLC control) (for reaction time, see Table 1). After removal of the solvent under reduced pressure the reaction mixture was added with $\mathrm{CH}_{2} \mathrm{Cl}_{2}$ and the undissolved urea filtered off. Removal of the solvent afforded the crude product which was purified by flash column chromatography on silica gel. 


\section{$N$-Benzylidene-benzylamine $N$-oxide (1a) ${ }^{2}$}

White solid. ${ }^{1} \mathrm{H}$ NMR (200 MHz, $\left.\mathrm{CDCl}_{3}\right)$ : $\delta$ 8.20-8.17 (m, $\left.2 \mathrm{H}\right), 7.49-7.38(\mathrm{~m}, 9 \mathrm{H}), 5.09$ (s, $2 \mathrm{H}$ ).

\section{$N$-Benzylidene- $n$-butylamine $N$-oxide $(1 \mathrm{~b})^{3}$}

Pale yellow solid. ${ }^{1} \mathrm{H}$ NMR (200 MHz, $\mathrm{CDCl}_{3}$ ): $\delta 8.24$ (m, $\left.2 \mathrm{H}\right), 7.44-7.39$ (m, $\left.4 \mathrm{H}\right), 3.97$ (t, $2 \mathrm{H}, J$ $=7.0 \mathrm{~Hz}$ ), 1.98 (quintet, $2 \mathrm{H}, J=7.0 \mathrm{~Hz}$ ), 1.43 (sextet, $2 \mathrm{H}, J=7.0 \mathrm{~Hz}$ ), 0.97 (t, $3 \mathrm{H}, J=7.0 \mathrm{~Hz}$ ).

\section{$N$-Benzylidene-phenylamine $N$-oxide (1c) ${ }^{4}$}

White solid. ${ }^{1} \mathrm{H}$ NMR (200 MHz, $\mathrm{CDCl}_{3}$ ): $\delta$ 8.42-8.37 (m, $\left.2 \mathrm{H}\right), 7.92$ (s, $\left.1 \mathrm{H}\right), 7.80-7.75(\mathrm{~m}, 2 \mathrm{H})$, 7.54-7.46 (m, $6 \mathrm{H})$.

\section{4-Nitrobenzylidene-benzylamine $N$-oxide (1d)}

$\mathrm{R}_{f}=0.30$ (petroleum ether-AcOEt 2:1). Yellow solid; m.p. 115-116 ${ }^{\circ} \mathrm{C} .{ }^{1} \mathrm{H}-\mathrm{NMR}(200 \mathrm{MHz}$, $\left.\mathrm{CDCl}_{3}\right): \delta(\mathrm{ppm})$ 8.38-8.32 (m, $\left.2 \mathrm{H}\right), 8.23-8.19(\mathrm{~m}, 2 \mathrm{H}), 7.54(\mathrm{~s}, 1 \mathrm{H}), 7.50-7.40(\mathrm{~m}, 5 \mathrm{H}), 5.11(\mathrm{~s}$, $2 \mathrm{H}) ;{ }^{13} \mathrm{C}-\mathrm{NMR}\left(50 \mathrm{MHz}, \mathrm{CDCl}_{3}\right): \delta$ (ppm) 147.4 (s), 135.8 (s), 132.3 (s), 132.0 (d), 129.2 (d, $3 \mathrm{C}$ ), 128.9 (d, 2 C), 128.6 (d, 2 C), 123.6 (d, 2 C), 72.0 (t); IR (KBr): 3113, 3071, 1596, 1562, 1514, 1341, 1152, 862, $714 \mathrm{~cm}^{-1}$; MS (70 eV): m/z (\%): 256 (13), 91 (100), 77 (8), 65 (36).

Anal. Calcd for $\mathrm{C}_{14} \mathrm{H}_{12} \mathrm{~N}_{2} \mathrm{O}_{3}$ : C, 65.62; H, 4.72; N, 10.93. Found: C, 65.26; H, 4.78; N, 10.98 .

\section{4-Nitrobenzylidene-butylamine $N$-oxide (1e)}

$\mathrm{R}_{f}=0.31$ (petroleum ether-AcOEt 3:2). Pale yellow solid; m.p. $78-79{ }^{\circ} \mathrm{C} .{ }^{1} \mathrm{H}-\mathrm{NMR}(200 \mathrm{MHz}$, $\left.\mathrm{CDCl}_{3}\right): \delta(\mathrm{ppm})$ 8.40-8.35 (m, $\left.2 \mathrm{H}\right), 8.24-8.20(\mathrm{~m}, 2 \mathrm{H}), 7.53(\mathrm{~s}, 1 \mathrm{H}), 4.00(\mathrm{t}, 2 \mathrm{H}, J=7.0 \mathrm{~Hz})$, 1.99 (quintet, $2 \mathrm{H}, J=7.0 \mathrm{~Hz}$ ), 1.44 (sextet, $2 \mathrm{H}, J=7.0 \mathrm{~Hz}$ ), 0.98 (t, $3 \mathrm{H}, J=7.0 \mathrm{~Hz}$ ); ${ }^{13} \mathrm{C}-\mathrm{NMR}$ (50 MHz, $\mathrm{CDCl}_{3}$ ): $\delta(\mathrm{ppm}) 147.4$ (s), 135.9 (s), 132.0 (d), 128.5 (d, 2 C), 123.6 (d, 2 C), 67.9 (t), 29.9 (t), 19.8 (t), 13.7 (q); IR $\left(\mathrm{CHCl}_{3}\right): 2964,2876,1599,1567,1521,1165 \mathrm{~cm}^{-1}$; MS (70 eV): $\mathrm{m} / \mathrm{z}$ (\%): 222 (30), 205 (20), 163 (82), 151 (34), 117 (40), 104 (22), 77 (64), 63 (56), 51 (100).

Anal. Calcd for $\mathrm{C}_{11} \mathrm{H}_{14} \mathrm{~N}_{2} \mathrm{O}_{3}$ : C, 59.45; H, 6.35; N, 12.60. Found: C, 59.44; H, 6.22; N, 12.69.

\section{2-Naphthylidene-benzylamine $N$-oxide (1f)}

$\mathrm{R}_{f}=0.43$ (petroleum ether-AcOEt 2:1). Yellow solid; m.p. 107-108 ${ }^{\circ} \mathrm{C}$. ${ }^{1} \mathrm{H}-\mathrm{NMR}(200 \mathrm{MHz}$, $\left.\mathrm{CDCl}_{3}\right): \delta(\mathrm{ppm}) 9.25(\mathrm{~s}, 1 \mathrm{H}), 7.92-7.77(\mathrm{~m}, 4 \mathrm{H}), 7.55-7.39(\mathrm{~m}, 8 \mathrm{H}), 5.09(\mathrm{~s}, 2 \mathrm{H}) ;{ }^{13} \mathrm{C}-\mathrm{NMR}(50$ $\mathrm{MHz}, \mathrm{CDCl}_{3}$ ): $\delta$ (ppm) 134.0 (d), 133.8 (s), 133.0 (s), 132.8 (s), 129.0 (d, 2 C), 128.9 (d), 128.7 (d, 3 C) 128.3 (d) 127.6 (d), 127.4 (s), 127.2 (d), 127.1 (d), 126.2 (d), 125.6 (d), 71.1 (t); IR (KBr): 
3056, 1595, 1572, 1497, 1451, 1354, 1161, 946, $731 \mathrm{~cm}^{-1}$; MS (70 eV): m/z (\%): 261 (45), 244 (4), 231 (8), 215 (2), 139 (23), 115 (23), 91 (100), 65 (44).

Anal. Calcd for $\mathrm{C}_{18} \mathrm{H}_{15} \mathrm{NO}$ : C, 82.73; H, 5.79; N, 5.36. Found: C, 82.69; H, 5.46; N, 5.44.

\section{2-Naphthylidene-butylamine $\mathrm{N}$-oxide (1g)}

$\mathrm{R}_{f}=0.29$ (petroleum ether-AcOEt 3:2). White solid; m.p. $42-43{ }^{\circ} \mathrm{C} .{ }^{1} \mathrm{H}-\mathrm{NMR}\left(200 \mathrm{MHz}, \mathrm{CDCl}_{3}\right): \delta$ (ppm) $9.26(\mathrm{~s}, 1 \mathrm{H}), 7.94-7.78(\mathrm{~m}, 4 \mathrm{H}), 7.55-7.45(\mathrm{~m}, 3 \mathrm{H}), 4.00$ (t, $2 \mathrm{H}, J=7.0 \mathrm{~Hz}), 2.03$ (quintet, $2 \mathrm{H}, J=7.0 \mathrm{~Hz}$ ), 1.45 (sextet, $2 \mathrm{H}, J=7.0 \mathrm{~Hz}), 0.99(\mathrm{t}, 3 \mathrm{H}, J=7.0 \mathrm{~Hz}) ;{ }^{13} \mathrm{C}-\mathrm{NMR}(50 \mathrm{MHz}$, $\mathrm{CDCl}_{3}$ ): $\delta$ (ppm) 134.0 (d), 133.8 (s), 132.9 (s), 128.9 (d), 128.2 (d), 127.7 (d), 127.5 (s), 127.2 (d), 127.0 (d), 126.2 (d), 125.6 (d), 67.0 (t), 29.8 (t), 19.8 (t), 13.7 (q); IR $\left(\mathrm{CHCl}_{3}\right):$ 3059, 2965, 2876, 1579, 1466, 1424, $1155 \mathrm{~cm}^{-1}$; MS (70 eV): m/z (\%): 227 (85), 210 (41), 168 (100), 141 (99), 128 (98), 115 (87), 84 (29).

Anal. Calcd for $\mathrm{C}_{15} \mathrm{H}_{17} \mathrm{NO}$ : C, 79.26; H, 7.54; N, 6.16. Found: C, 79.24; H, 7.91; N, 6.16.

\section{3-Methoxybenzylidene-benzylamine $N$-oxide (1h)}

$\mathrm{R}_{f}=0.33$ (petroleum ether-AcOEt 3:2). Pale yellow solid; m.p. 40-42 ${ }^{\circ} \mathrm{C} .{ }^{1} \mathrm{H}-\mathrm{NMR}(200 \mathrm{MHz}$, $\left.\mathrm{CDCl}_{3}\right): \delta(\mathrm{ppm}) 8.19$ (br s, $\left.1 \mathrm{H}\right), 7.52-7.30(\mathrm{~m}, 8 \mathrm{H}), 7.00-6.95(\mathrm{~m}, 1 \mathrm{H}), 5.08(\mathrm{~s}, 2 \mathrm{H}), 3.83(\mathrm{~s}, 3$ $\mathrm{H}) ;{ }^{13} \mathrm{C}-\mathrm{NMR}\left(50 \mathrm{MHz}, \mathrm{CDCl}_{3}\right.$ ): $\delta$ (ppm) 159.1 (s), 134.0 (d), 133.0 (s), 131.3 (s), 128.9 (d, 3 C), 128.6 (d, 3 C), 121.4 (d), 117.2 (d), 112.1 (d), 71.1 (t), 55.2 (q); IR $\left(\mathrm{CHCl}_{3}\right)$ : 3052, 2943, 1703, 1595, 1466, 1269, 1174, $1040 \mathrm{~cm}^{-1}$; MS (70 eV): m/z (\%): 241 (41), 224 (7), 211 (8), 135 (4), 91 (100), 65 (53), 51 (26).

Anal. Calcd for $\mathrm{C}_{15} \mathrm{H}_{15} \mathrm{NO}_{2}$ : C, 74.67; H, 6.27; N, 5.81. Found: C, 74.62; H, 6.63; N, 5.89.

\section{3-Methoxybenzylidene-4-methoxybenzylamine $N$-oxide (1i)}

$\mathrm{R}_{f}=0.28$ (petroleum ether-AcOEt 3:1). Colorless oil; ${ }^{1} \mathrm{H}-\mathrm{NMR}\left(200 \mathrm{MHz}, \mathrm{CDCl}_{3}\right): \delta(\mathrm{ppm}) 8.19(\mathrm{~m}$, $1 \mathrm{H}), 7.44-7.22(\mathrm{~m}, 5 \mathrm{H}), 6.97-6.87(\mathrm{~m}, 3 \mathrm{H}), 4.96(\mathrm{~s}, 2 \mathrm{H}), 3.80(\mathrm{~s}, 6 \mathrm{H}) ;{ }^{13} \mathrm{C}-\mathrm{NMR}(50 \mathrm{MHz}$, $\mathrm{CDCl}_{3}$ ): $\delta$ (ppm) 159.8 (s), 159.1 (s), 133.6 (d), 131.3 (s), 130.6 (d, 2C), 128.9 (d), 124.9 (s), 121.4 (d), 117.1 (d), 114.1 (d, 2 C), 112.0 (d), 70.5 (t), 55.2 (q, 2 C); IR $\left(\mathrm{CHCl}_{3}\right)$ : 3083, 3004, 2966, 1590, 1613, 1515, 1466, 1253, 1176, $1037 \mathrm{~cm}^{-1}$; MS (70 eV): m/z (\%): 271 (20), 255 (5), 121 (100), 91 (20), 77 (37).

Anal. Calcd for $\mathrm{C}_{16} \mathrm{H}_{17} \mathrm{NO}_{3}$ : C, 70.83; H, 6.32; N, 5.16. Found: C, 70.75; H, 6.69; N, 4.76. 


\section{3-Methoxybenzylidene-butylamine $N$-oxide (1j)}

$\mathrm{R}_{f}=0.36$ (AcOEt-petroleum ether 2:1). Pale yellow oil. ${ }^{1} \mathrm{H}-\mathrm{NMR}\left(200 \mathrm{MHz}, \mathrm{CDCl}_{3}\right.$ ): $\delta$ (ppm) 8.22 (m, $1 \mathrm{H}), 7.51-7.47$ (m, $1 \mathrm{H}), 7.37(\mathrm{~s}, 1 \mathrm{H}), 7.31$ (m, $1 \mathrm{H}), 7.01-6.95$ (m, $1 \mathrm{H}), 3.94$ (t, $2 \mathrm{H}, J=7.0$ $\mathrm{Hz}$ ), 3.85 (s, $3 \mathrm{H}$ ), 1.99 (quintet, $2 \mathrm{H}, J=7.0 \mathrm{~Hz}$ ), 1.43 (sextet, $2 \mathrm{H}, J=7.0 \mathrm{~Hz}$ ), 0.98 (t, $3 \mathrm{H}, J=7.0$ $\mathrm{Hz}$ ); ${ }^{13} \mathrm{C}-\mathrm{NMR}\left(50 \mathrm{MHz}, \mathrm{CDCl}_{3}\right.$ ): $\delta$ (ppm) 159.2 (s), 134.0 (d), 131.5 (s), 129.0 (d), 121.3 (d), 117.0 (d), 112.1 (d), 67.0 (t), 55.2 (q), 29.7 (t), 19.7 (t), 13.6 (q); IR $\left(\mathrm{CHCl}_{3}\right): 2964,2876,1703$, 1591, 1466, 1431, 1270, 1176, 1151, $1040 \mathrm{~cm}^{-1}$; MS (70 eV): m/z (\%): 207 (68), 190 (39), 175 (5), 162 (9), 148 (100), 134 (49), 121 (41), 108 (51), 91 (29), 77 (47), 65 (29), 51 (49).

Anal. Calcd for $\mathrm{C}_{12} \mathrm{H}_{17} \mathrm{NO}_{2}$ : C, 69.54; H, 8.27; N, 6.76. Found: C, 69.18; H, 8.13; N, 7.12.

\section{4-Methoxybenzylidene-benzylamine $N$-oxide (1k)}

$\mathrm{R}_{f}=0.22$ (AcOEt-petroleum ether 3:2). White solid; m.p. 96-97 ${ }^{\circ} \mathrm{C} .{ }^{1} \mathrm{H}-\mathrm{NMR}\left(200 \mathrm{MHz}, \mathrm{CDCl}_{3}\right): \delta$ (ppm) 8.20 (m, $2 \mathrm{H}), 7.49-7.38(\mathrm{~m}, 5 \mathrm{H}), 7.33$ (s, $1 \mathrm{H}), 6.91(\mathrm{~m}, 2 \mathrm{H}), 5.05(\mathrm{~s}, 2 \mathrm{H}), 3.84(\mathrm{~s}, 3 \mathrm{H})$; ${ }^{13} \mathrm{C}-\mathrm{NMR}\left(50 \mathrm{MHz}, \mathrm{CDCl}_{3}\right.$ ): $\delta$ (ppm) 160.8 (s), 133.7 (d), 133.2 (s), 130.3 (d, $\left.2 \mathrm{C}\right), 128.9$ (d, 2 C) 128.6 (d, 2 C), 128.5 (d), 123.2 (s), 113.5 (d, 2 C), 70.6 (t), 55.2 (q); IR $\left(\mathrm{CHCl}_{3}\right)$ : 3052, 2938, 2841, 1604, 1508, 1258, 1172, $1031 \mathrm{~cm}^{-1}$; MS (70 eV): m/z (\%): 241 (41), 224 (7), 224 (5), 211 (5), 135 (8), 91 (100), 77 (20), 65 (40), 51 (30).

Anal. Calcd for $\mathrm{C}_{15} \mathrm{H}_{15} \mathrm{NO}_{2}$ : C, 74.67; H, 6.27; N, 5.81. Found: C, 74.49; H, 6.21; N, 5.76.

\section{4-Methoxybenzylidene-4-methoxybenzylamine $N$-oxide $(11)^{5}$}

Pale yellow solid; ${ }^{1} \mathrm{H}$ NMR (200 MHz, $\mathrm{CDCl}_{3}$ ): $\delta 8.19$ (m, $\left.2 \mathrm{H}\right), 7.40$ (m, $\left.2 \mathrm{H}\right), 7.26$ (s, $\left.1 \mathrm{H}\right), 6.91$ (m, 4 H), 4.96 (s, 2 H), 3.83 (s, 3 H), $3.82(\mathrm{~s}, 3 \mathrm{H})$.

\section{4-Methoxybenzylidene-butylamine $N$-oxide (1m)}

$\mathrm{R}_{f}=0.25$ (AcOEt-petroleum ether 3:1). Pale yellow oil. ${ }^{1} \mathrm{H}-\mathrm{NMR}\left(200 \mathrm{MHz}, \mathrm{CDCl}_{3}\right.$ ): $\delta$ (ppm) 8.23 (m, $2 \mathrm{H}), 7.31(\mathrm{~s}, 1 \mathrm{H}), 6.93(\mathrm{~m}, 2 \mathrm{H}), 3.90$ (t, $2 \mathrm{H}, J=7.0 \mathrm{~Hz}), 3.84(\mathrm{~s}, 3 \mathrm{H}), 1.98$ (quintet, $2 \mathrm{H}, J=$ $7.0 \mathrm{~Hz}$ ), 1.41 (sextet, $2 \mathrm{H}, J=7.0 \mathrm{~Hz}), 0.97(\mathrm{t}, 3 \mathrm{H}, J=7.0 \mathrm{~Hz}) ;{ }^{13} \mathrm{C}-\mathrm{NMR}\left(50 \mathrm{MHz}, \mathrm{CDCl}_{3}\right): \delta$ (ppm) 160.6 (s), 133.7 (d), 130.2 (d, 2 C), 123.3 (s), 113.6 (d, 2 C), 66.4 (t), 55.2 (q), 29.7 (t), 19.8 (t), 13.6 (q); IR $\left(\mathrm{CHCl}_{3}\right): 2964,2936,2875,1604,1508,1464,1426,1269,1172,1156,1031 \mathrm{~cm}^{-1}$; MS (70 eV): m/z (\%): 207 (27), 190 (9), 162 (4), 148 (75), 134 (24), 121 (84), 108 (100), 91 (21), 77 (32).

Anal. Calcd for $\mathrm{C}_{12} \mathrm{H}_{17} \mathrm{NO}_{2}$ : C, 69.54; H, 8.27; N, 6.76. Found: C, 69.24; H, 8.17; N, 6.73. 


\section{(E)-3-Phenylpropenylidene-benzylamine $N$-oxide (1n)}

$\mathrm{R}_{f}=0.27$ (AcOEt-petroleum ether 3:2). White solid; m.p. 126-127 ${ }^{\circ} \mathrm{C} .{ }^{1} \mathrm{H}-\mathrm{NMR}\left(200 \mathrm{MHz}, \mathrm{CDCl}_{3}\right)$ : $\delta(\mathrm{ppm}) 7.52-7.19(\mathrm{~m}, 12 \mathrm{H}), 6.94(\mathrm{~d}, 1 \mathrm{H}, J=16.1 \mathrm{~Hz}), 4.96(\mathrm{~s}, 2 \mathrm{H}) ;{ }^{13} \mathrm{C}-\mathrm{NMR}\left(50 \mathrm{MHz}, \mathrm{CDCl}_{3}\right)$ : $\delta(\mathrm{ppm}) 138.1$ (d), 136.2 (d), 135.7 (s), 132.7 (s), 128.9 (d, 2 C), 128.8 (d), 128.7 (d, 2 C), 128.5 (d, 3 C), 127.0 (d, 2 C), 118.1 (d), 69.1 (t); IR (KBr): 3086, 3051, 2998, 1546, 1457, 1424, 1200, 1122 , 961, 747, $701 \mathrm{~cm}^{-1}$; MS (70 eV): m/z (\%): 237 (40), 220 (6), 160 (13), 146(6), 130 (3), 115 (21), 105 (7), 91 (100), 77 (14), 65 (41).

Anal. Calcd for $\mathrm{C}_{16} \mathrm{H}_{15} \mathrm{NO}$ : C, 80.98; H, 6.37; N, 5.90. Found: C, 80.98; H, 6.65; N, 5.55.

\section{$N$-Benzylidene-(R)- $\alpha$-methylbenzylamine $N$-oxide (10) ${ }^{6}$}

Colorless oil. ${ }^{1} \mathrm{H}$ NMR (200 MHz, $\left.\mathrm{CDCl}_{3}\right): \delta 8.24-8.19$ (m, $\left.2 \mathrm{H}\right), 7.53-7.38(\mathrm{~m}, 9 \mathrm{H}), 5.18(\mathrm{q}, 1 \mathrm{H}$, $J=6.9 \mathrm{~Hz}), 1.87(\mathrm{~d}, 3 \mathrm{H}, J=6.9 \mathrm{~Hz})$.

$[\alpha]_{\mathrm{D}}^{21}=-48.8\left(\mathrm{c} 1.0, \mathrm{CHCl}_{3}\right)$.

\section{$N$-n-Butylidene-benzylamine $N$-oxide (1p) ${ }^{7}$}

Colorless oil. ${ }^{1} \mathrm{H}$ NMR (200 MHz, $\left.\mathrm{CDCl}_{3}\right): \delta 7.35$ (m, $\left.5 \mathrm{H}\right), 6.63(\mathrm{t}, 1 \mathrm{H}, J=5.8 \mathrm{~Hz}), 4.85(\mathrm{~s}, 2 \mathrm{H})$, 2.43 (q, $2 \mathrm{H}, J=6.9 \mathrm{~Hz}$ ), 1.48 (sextet, $2 \mathrm{H}, J=7.3 \mathrm{~Hz}$ ), 0.90 (t, $3 \mathrm{H}, J=7.3 \mathrm{~Hz}$ ).

\section{$N$-2,2-Dimethylpropylidene-benzylamine $N$-oxide (1q)}

$\mathrm{R}_{f}=0.25$ (petroleum ether-AcOEt 1:1). White solid; m.p. 65-67 ${ }^{\circ} \mathrm{C} .{ }^{1} \mathrm{H}$ NMR $\left(200 \mathrm{MHz}, \mathrm{CDCl}_{3}\right): \delta$ $7.41(\mathrm{~m}, 5 \mathrm{H}), 6.55(\mathrm{~s}, 1 \mathrm{H}), 4.87(\mathrm{~s}, 2 \mathrm{H}), 1.28(\mathrm{~s}, 9 \mathrm{H}) ;{ }^{13} \mathrm{C} \mathrm{NMR}\left(50 \mathrm{MHz}, \mathrm{CDCl}_{3}\right): \delta 145.1(\mathrm{~d})$, 133.4 (s), 128.8 (d, 2 C), 128.7 (d, 2 C), 128.6 (d), 70.5 (t), 32.9 (s), 26.0 (q, 3 C); IR $\left(\mathrm{CHCl}_{3}\right)$ : 2991, 2960, 2865, 1592, 1497, 1455, 1361, $1142 \mathrm{~cm}^{-1}$; MS (70 eV): m/z (\%): 191 (1), 174 (6), 160 (6), 132(3), 91 (100), 57 (19).

Anal. Calcd for $\mathrm{C}_{12} \mathrm{H}_{17} \mathrm{NO}$ : C, 75.35; H, 8.96; N, 7.32. Found: C, 75.64; H, 8.98; N, 7.63.

\section{4-Methoxybenzylidene-1,3,4,6-tetra- $O$-acetyl-2-glucopyranosylamine $\mathrm{N}$-oxide (1r)}

$\mathrm{R}_{f}=0.35$ (AcOEt-petroleum ether 2:1). White solid; $198-199{ }^{\circ} \mathrm{C}$ (dec.). ${ }^{1} \mathrm{H}$ NMR $\left(200 \mathrm{MHz}, \mathrm{CDCl}_{3}\right)$ : $\delta 8.21(\mathrm{~m}, 2 \mathrm{H}), 7.26(\mathrm{~s}, 1 \mathrm{H}), 6.93(\mathrm{~m}, 2 \mathrm{H}), 6.21(\mathrm{~d}, 1 \mathrm{H}, J=8.2 \mathrm{~Hz}), 5.94(\mathrm{t}, 1 \mathrm{H}, J=9.7 \mathrm{~Hz})$, $5.18(\mathrm{t}, 1 \mathrm{H}, J=9.7 \mathrm{~Hz}), 4.38(\mathrm{dd}, 1 \mathrm{H}, J=12.6,4.4 \mathrm{~Hz}), 4.16-4.02(\mathrm{~m}, 2 \mathrm{H}), 3.97$ (ddd, $1 \mathrm{H}, J=$ 10.0, 4.4, $2.1 \mathrm{~Hz}), 3.84(\mathrm{~s}, 3 \mathrm{H}), 2.08(\mathrm{~s}, 3 \mathrm{H}), 2.04(\mathrm{~s}, 6 \mathrm{H}), 1.91(\mathrm{~s}, 3 \mathrm{H}) ;{ }^{13} \mathrm{C} \mathrm{NMR}(50 \mathrm{MHz}$, $\mathrm{CDCl}_{3}$ ): $\delta 170.4$ (s), 169.8 (s), 169.0 (s), 167.9 (s), 161.5 (s), 136.1 (d), 130.9 (d, 2 C), 122.4 (s), 113.9 (d, 2 C), 90.7 (d), 75.7 (d), 72.4 (d), 70.4 (d), 68.1 (d), 61.4 (t), 55.4 (q), 20.8 (q), 20.7 (q), 20.6 (q), 20.5 (q); IR $\left(\mathrm{CHCl}_{3}\right): 3018,2961,2841,1756,1604,1508,1368,1224,1210,1074 \mathrm{~cm}^{-1}$; 
MS (70 eV): m/z (\%): 481 (11), 422 (6), 362 (2), 229 (12), 211 (17), 194 (18), 169 (28), 152 (100), $134(31), 109(23)$.

$[\alpha]_{\mathrm{D}}^{22}=+91.3\left(\mathrm{c} 0.27, \mathrm{CHCl}_{3}\right)$.

Anal. Calcd for $\mathrm{C}_{22} \mathrm{H}_{27} \mathrm{NO}_{11}$ : C, 54.88; H, 5.65; N, 2.91. Found: C, 54.84; H, 6.05; N, 2.68.

\section{2-Methyl-1-pyrroline $N$-oxide (1s) ${ }^{8}$}

Colorless oil. ${ }^{1} \mathrm{H}$ NMR (200 MHz, $\mathrm{CDCl}_{3}$ ): $\delta 3.96$ (m, $\left.2 \mathrm{H}\right), 2.70$ (m, $\left.2 \mathrm{H}\right), 2.15-2.00$ (m, $\left.5 \mathrm{H}\right)$.

\section{(3S,4S)-3,4-O-tert-Butyldimethylsilyl-1-pyrroline $\mathrm{N}$-oxide (1t) ${ }^{9}$}

Colorless oil. ${ }^{1} \mathrm{H}$ NMR (200 MHz, $\mathrm{CDCl}_{3}$ ): $\delta 6.85$ (q, $1 \mathrm{H}, J=1.7 \mathrm{~Hz}$ ), 4.67 (br s, $\left.1 \mathrm{H}\right), 4.72-4.60$ (m, $1 \mathrm{H}), 4.31-4.20(\mathrm{~m}, 2 \mathrm{H}), 0.87(\mathrm{~s}, 18 \mathrm{H}), 0.10(\mathrm{~s}, 3 \mathrm{H}), 0.09(\mathrm{~s}, 3 \mathrm{H}), 0.08(\mathrm{~s}, 3 \mathrm{H}), 0.06(\mathrm{~s}, 3 \mathrm{H})$.

\section{2-Methyl-tert-butyldimethylsilyloxy-1-pyrroline $N$-oxide (1u)}

$\mathrm{R}_{f}=0.25\left(\mathrm{CH}_{2} \mathrm{Cl}_{2}-\mathrm{MeOH} 25: 1\right)$. Colorless oil. ${ }^{1} \mathrm{H} \mathrm{NMR}\left(400 \mathrm{MHz}, \mathrm{CDCl}_{3}\right): \delta 4.60(\mathrm{~m}, 2 \mathrm{H}), 4.02$ $(\mathrm{m}, 2 \mathrm{H}), 2.84(\mathrm{~m}, 2 \mathrm{H}), 2.18-2.10(\mathrm{~m}, 2 \mathrm{H}), 0.89(\mathrm{~s}, 9 \mathrm{H}), 0.08(\mathrm{~s}, 6 \mathrm{H}) ;{ }^{13} \mathrm{C} \mathrm{NMR}(50 \mathrm{MHz}$, $\left.\mathrm{CDCl}_{3}\right): \delta 148.4(\mathrm{~s}), 62.5(\mathrm{t}), 58.5(\mathrm{t}), 29.2(\mathrm{t}), 25.5(\mathrm{q}, 3 \mathrm{C}), 17.9(\mathrm{~s}), 16.9(\mathrm{t}),-5.7(\mathrm{q}, 2 \mathrm{C}) ; \mathrm{IR}$ $\left(\mathrm{CHCl}_{3}\right): 2956,2930,2885,2858,1690,1619,1472,1260,1210,1122,1006 \mathrm{~cm}^{-1}$; MS (70 eV): m/z (\%): 229 (1), 214 (17), 172 (100), 156 (42), 129 (87), 115 (10), 101 (41), 75 (90), 59 (60).

Anal. Calcd for $\mathrm{C}_{11} \mathrm{H}_{23} \mathrm{NO}_{2} \mathrm{Si}$ : C, 57.59; H, 10.11; N, 6.11. Found: C, 57.53; H, 10.30; N, 6.23.

\section{(5S)-5-Methyl-tert-butyldimethylsilyloxy-1-pyrroline $N$-oxide (1v)}

$\mathrm{R}_{f}=0.25\left(\mathrm{CH}_{2} \mathrm{Cl}_{2}-\mathrm{MeOH} 25: 1\right)$. Colorless oil. ${ }^{1} \mathrm{H}$ NMR (400 MHz, $\left.\mathrm{CDCl}_{3}\right): \delta 6.95$ (br s, $\left.1 \mathrm{H}\right), 4.31$ (dd, $1 \mathrm{H}, J=10.9,2.8 \mathrm{~Hz}), 4.01(\mathrm{~m}, 1 \mathrm{H}), 3.07$ (ddd, $1 \mathrm{H}, J=10.9,2.6,0.5 \mathrm{~Hz}), 2.73-2.56(\mathrm{~m}, 2 \mathrm{H})$, 2.38-2.24 (m, $2 \mathrm{H}), 0.80(\mathrm{~s}, 9 \mathrm{H}), 0.08(\mathrm{~s}, 3 \mathrm{H}), 0.07$ (s, $3 \mathrm{H}) ;{ }^{13} \mathrm{C} \mathrm{NMR}\left(50 \mathrm{MHz}, \mathrm{CDCl}_{3}\right): \delta 135.1$ (d), $73.9(\mathrm{~d}), 61.5(\mathrm{t}), 27.5$ (t), 25.8 (q, 3 C), $21.3(\mathrm{t}), 18.2(\mathrm{~s}),-5.4(\mathrm{q}),-5.5(\mathrm{q})$; IR $\left(\mathrm{CHCl}_{3}\right): 2956$, 2930, 2884, 2857, 1595, 1472, 1462, 1254, $1125 \mathrm{~cm}^{-1}$; MS (70 eV): m/z (\%): 229 (1), 214 (16), 198 (4), 172 (100), 156 (38), 115 (22), 75 (93).

$[\alpha]_{\mathrm{D}}^{22}=-7.2\left(\mathrm{c} 0.50, \mathrm{CHCl}_{3}\right)$.

Anal. Calcd for $\mathrm{C}_{11} \mathrm{H}_{23} \mathrm{NO}_{2} \mathrm{Si}$ : C, 57.59; H, 10.11; N, 6.11. Found: C, 57.26; H, 10.46; N, 5.93. 


\section{References}

1. Davis, B. G.; Maughan, M. A. T.; Chapman, T. M.; Villard, R.; Courtney, S. Org. Lett. 2002, 4, 103.

2. Goti, A.; Cardona, F.; Soldaini, G. Org. Synth. 2005, 81, 204.

3. Bartoli, G.; Marcantoni, E.; Petrini, M. J. Org. Chem. 1992, 57, 5834.

4. Murray, R. W.; Iyanar, K.; Chen, J.; Wearing, J. T. J. Org. Chem. 1996, 61, 8099.

5. Yamada, Y. M. A.; Tabata, H.; Ichinohe, M.; Takahashi, H.; Ikegami, S. Tetrahedron 2004, 60, 4087.

6. Jost, S.; Gimbert, Y.; Greene, A. E.; Fotiadu, F. J. Org. Chem. 1997, 62, 6672.

7. Dondoni, A.; Franco, S; Junquera, F.; Merchan, F.; Merino, P.; Tejero, T. Synth. Commun. 1994, 24, 2537.

8. Saladino, R.; Neri, V.; Cardona, F.; Goti, A. Adv. Synth. Catal. 2004, 346, 639.

9. Goti, A.; Cardona, F.; Brandi, A.; Picasso, S.; Vogel, P. Tetrahedron: Asymmetry 1996, 7, 1659. 

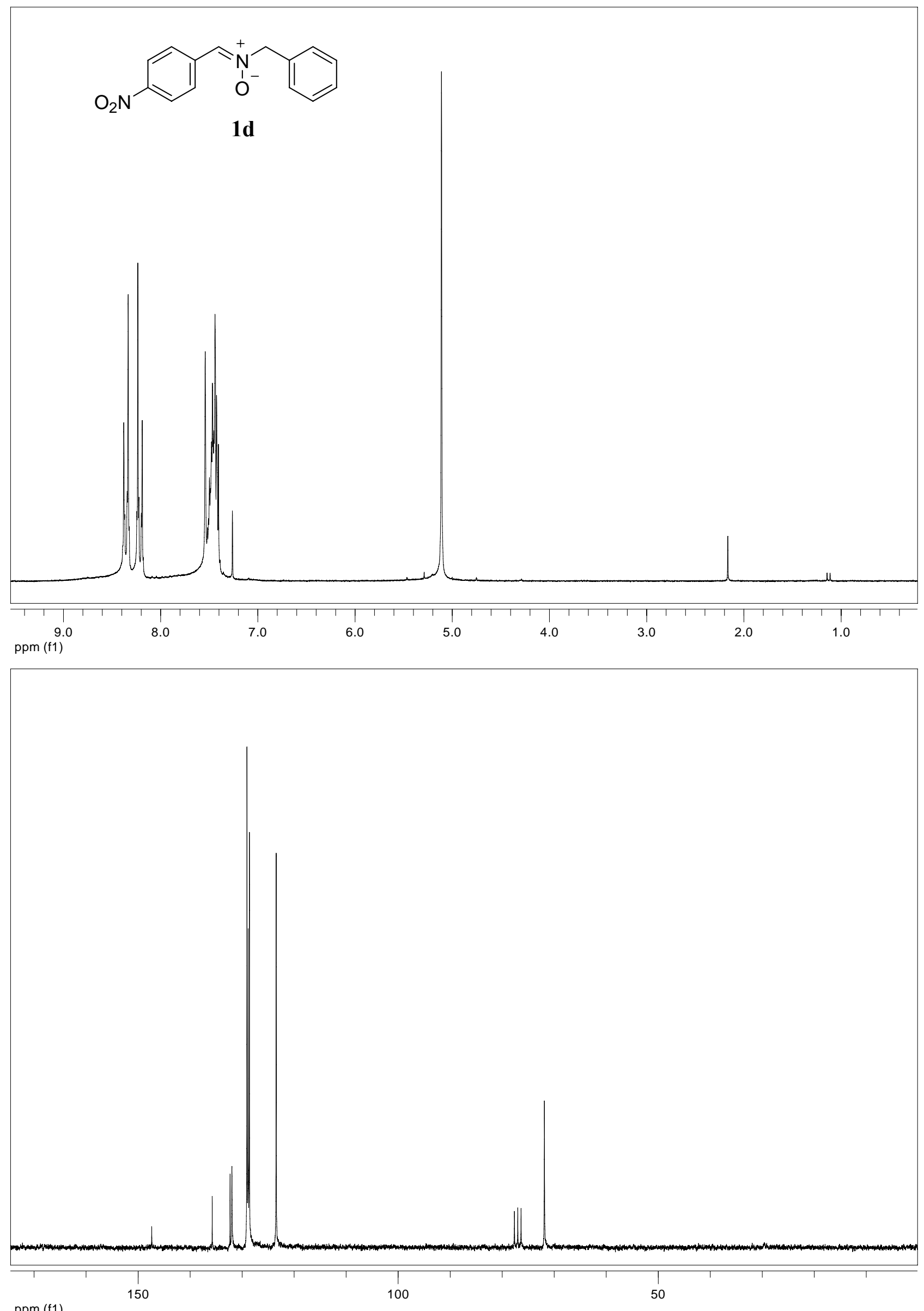


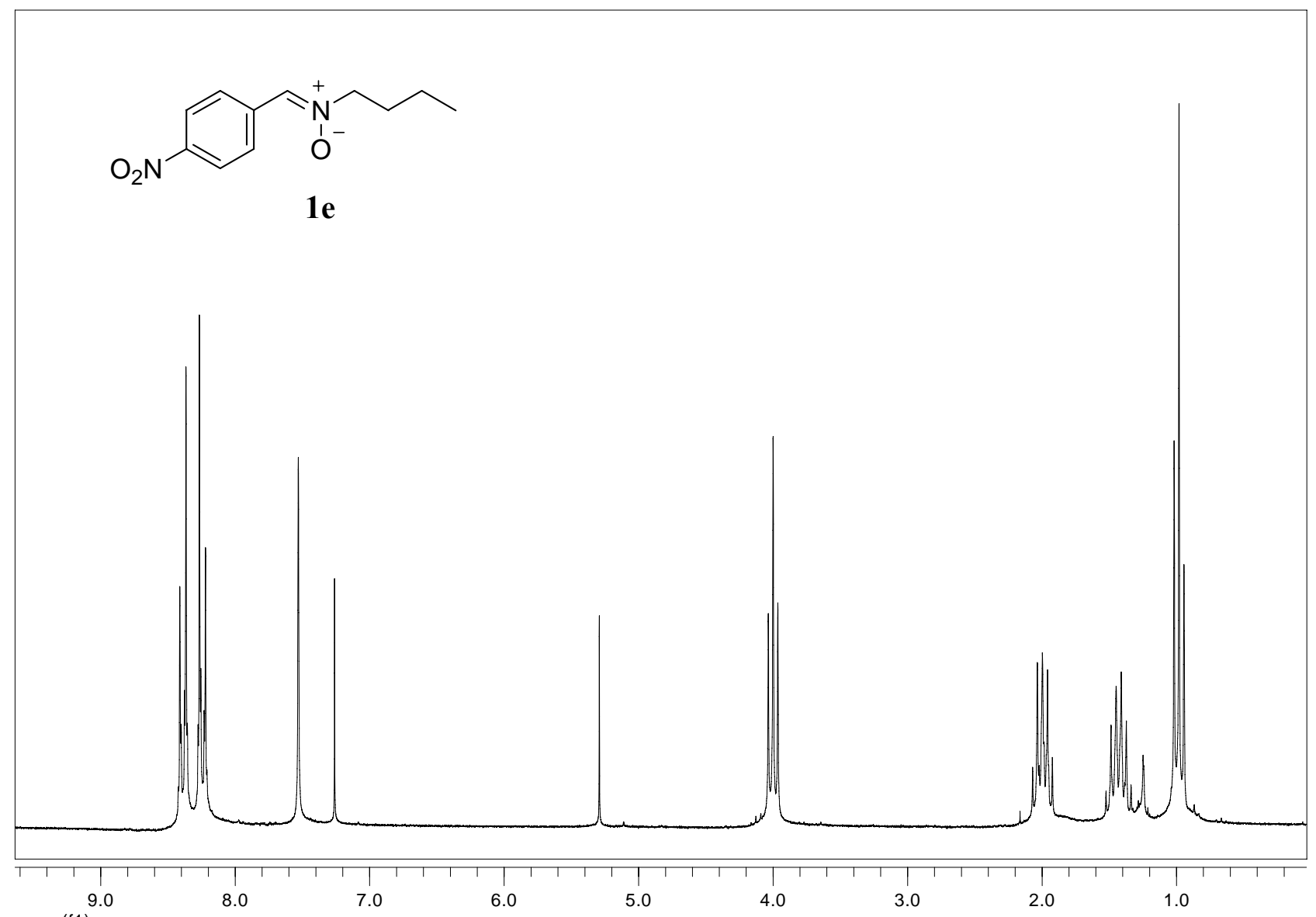

ppm (f1)

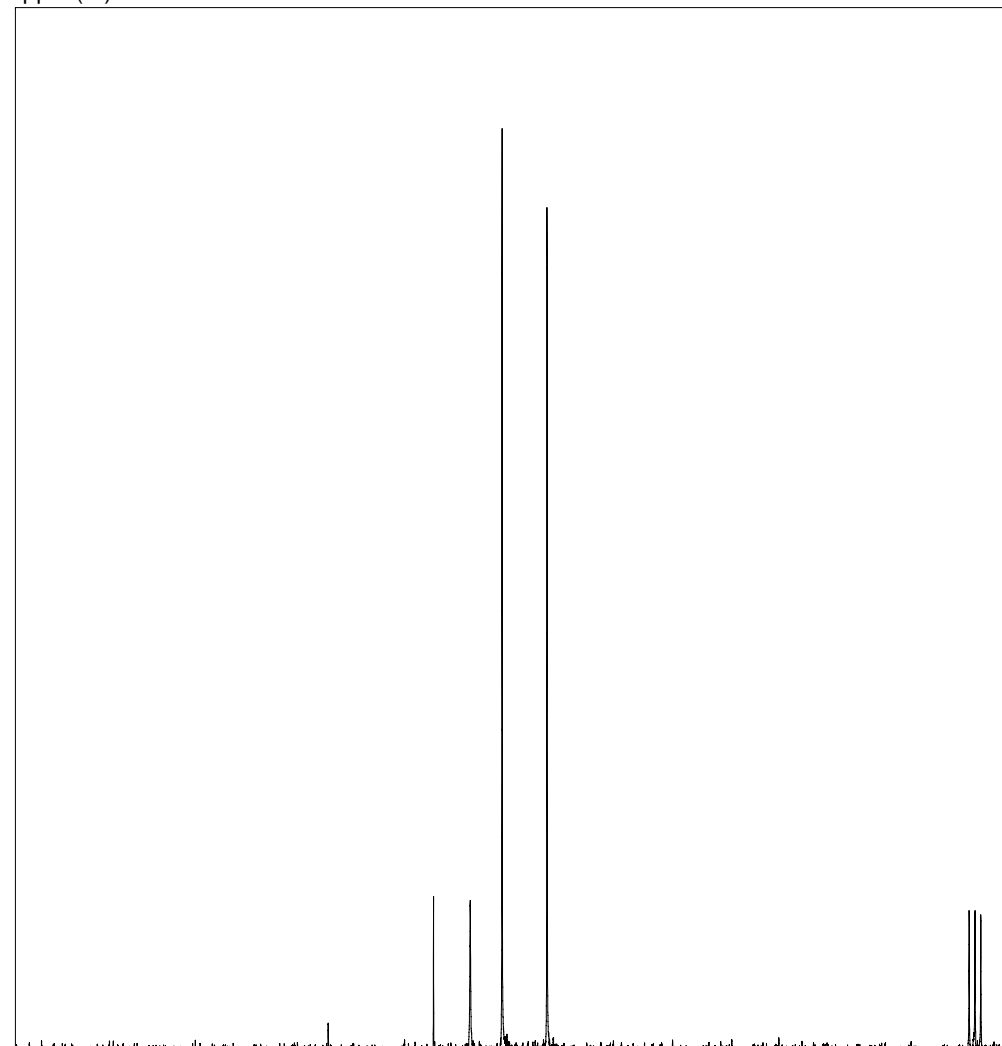

1.

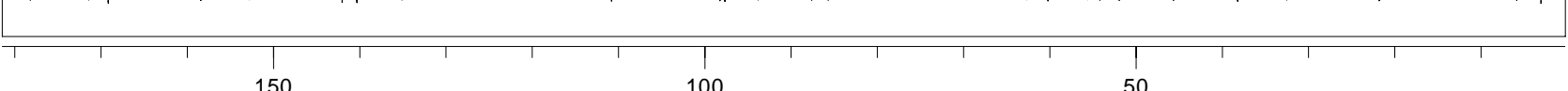

ppm (f1) 


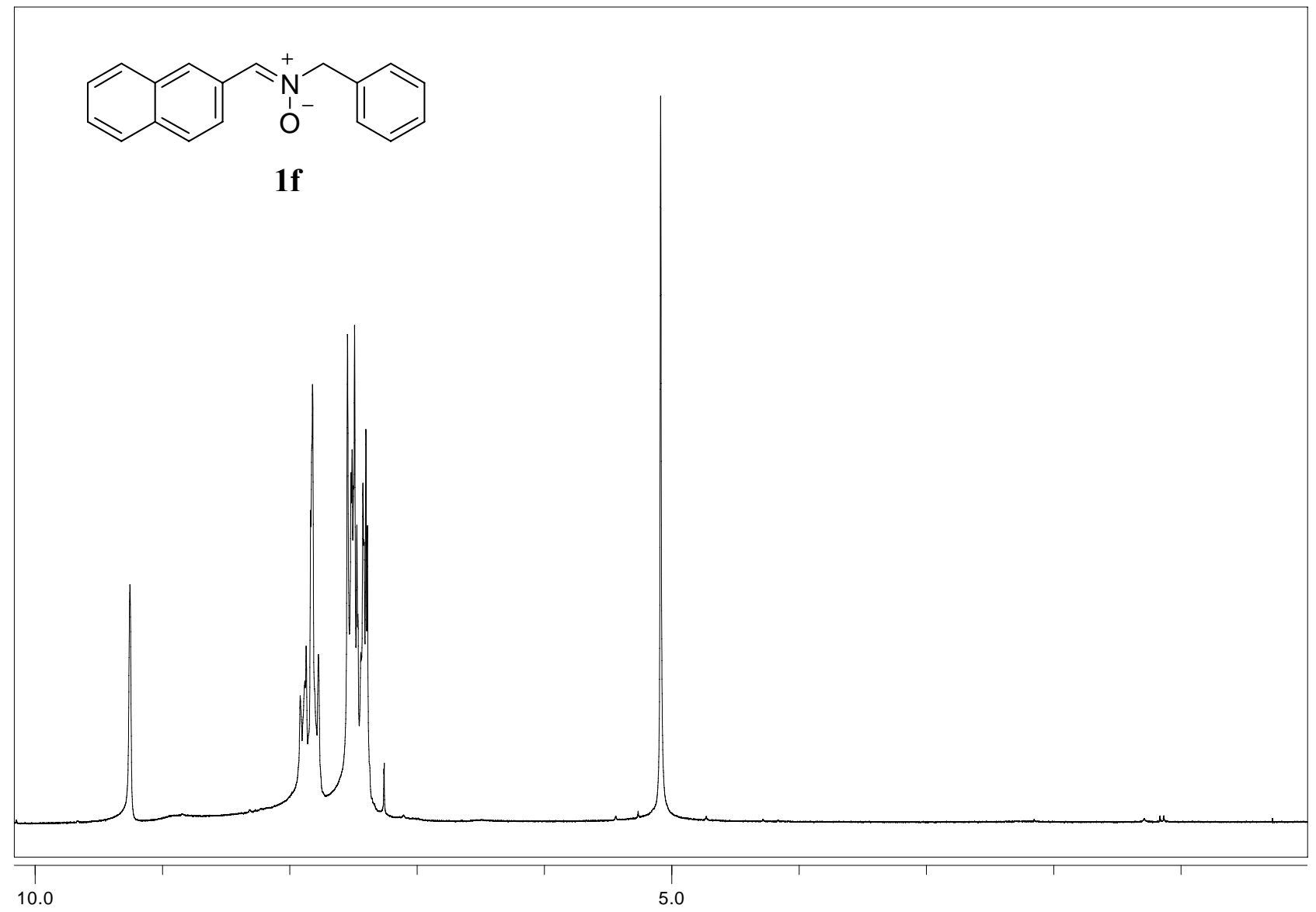

ppm (f1)

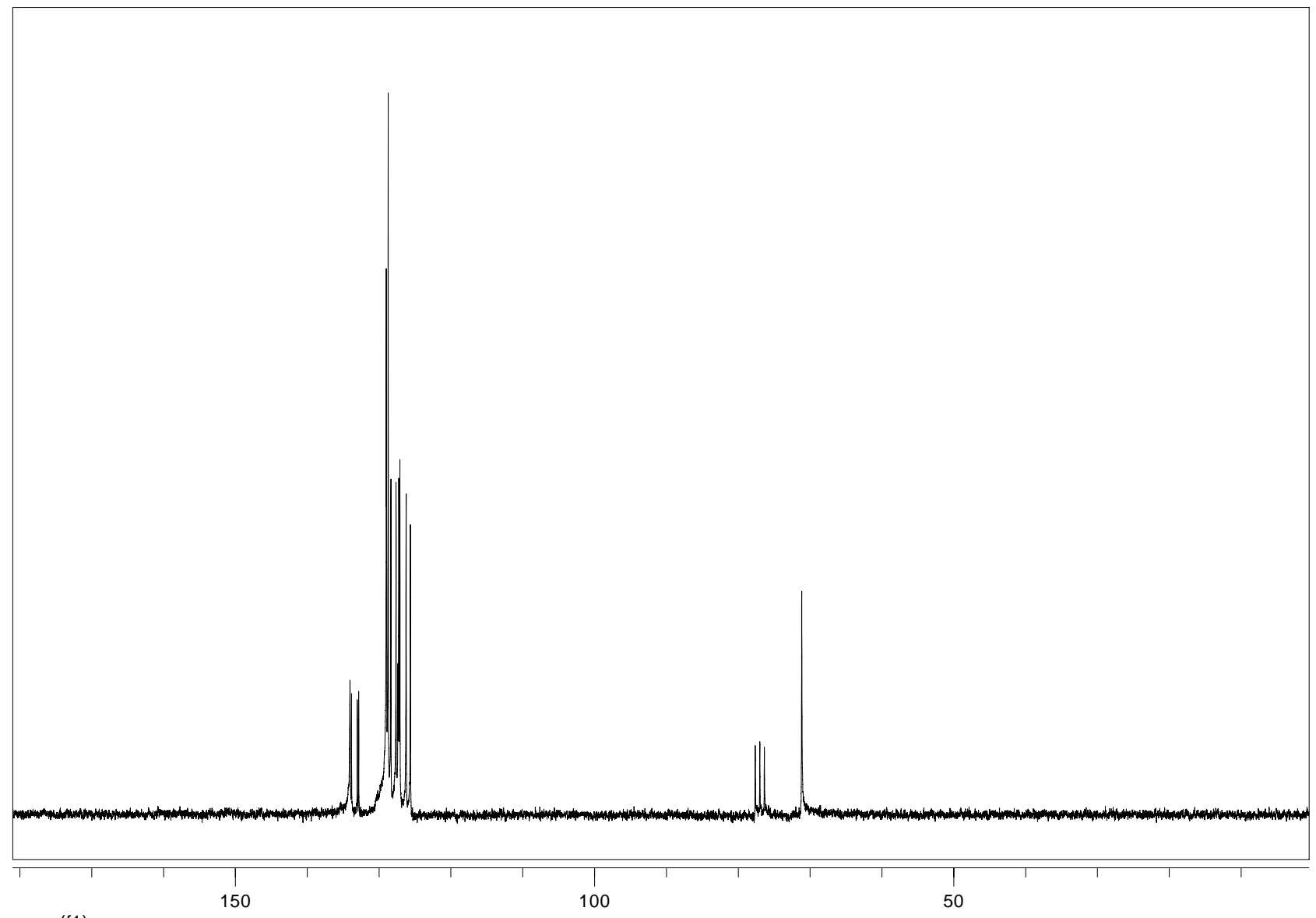

ppm (f1) 


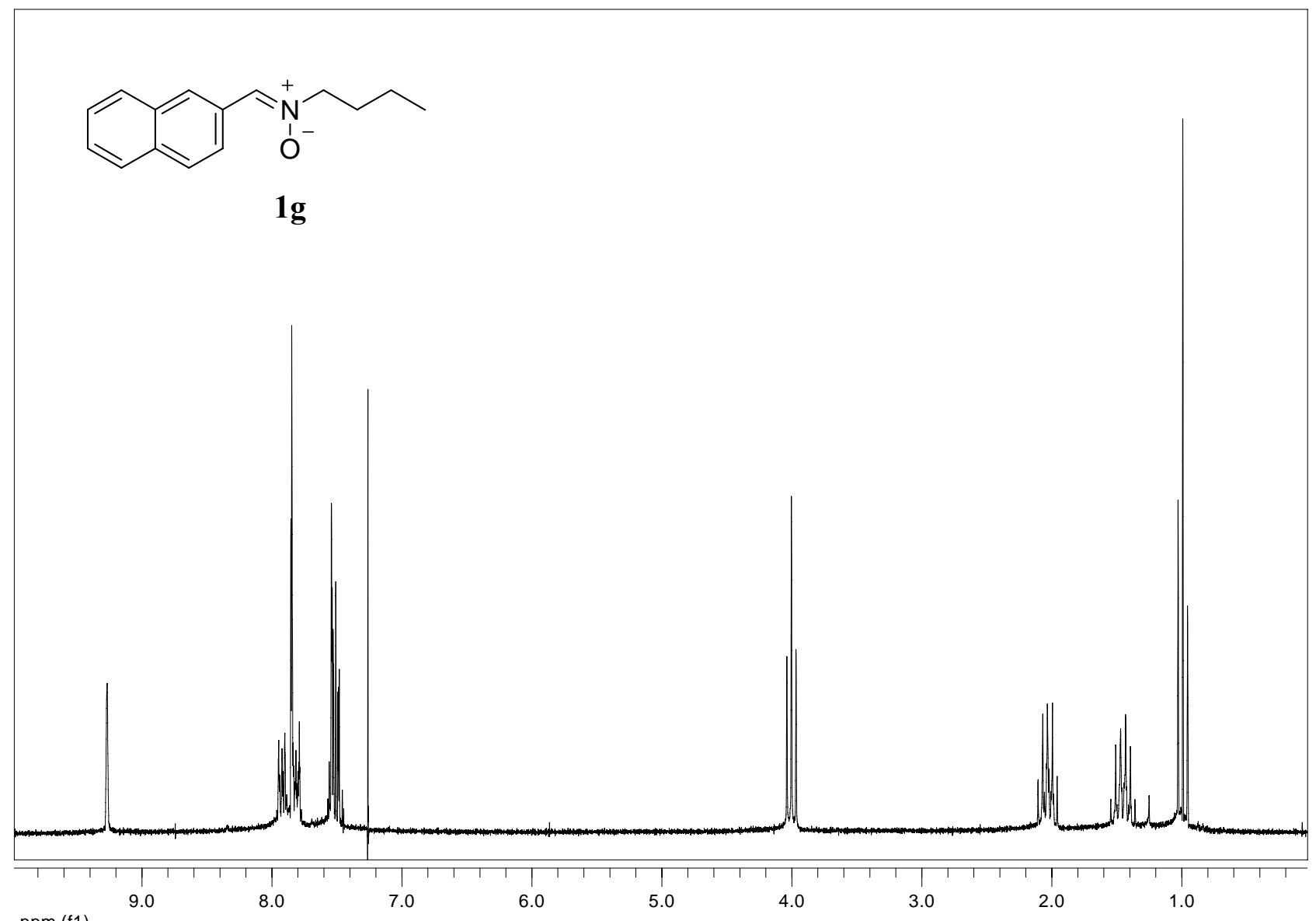

ppm (f1)

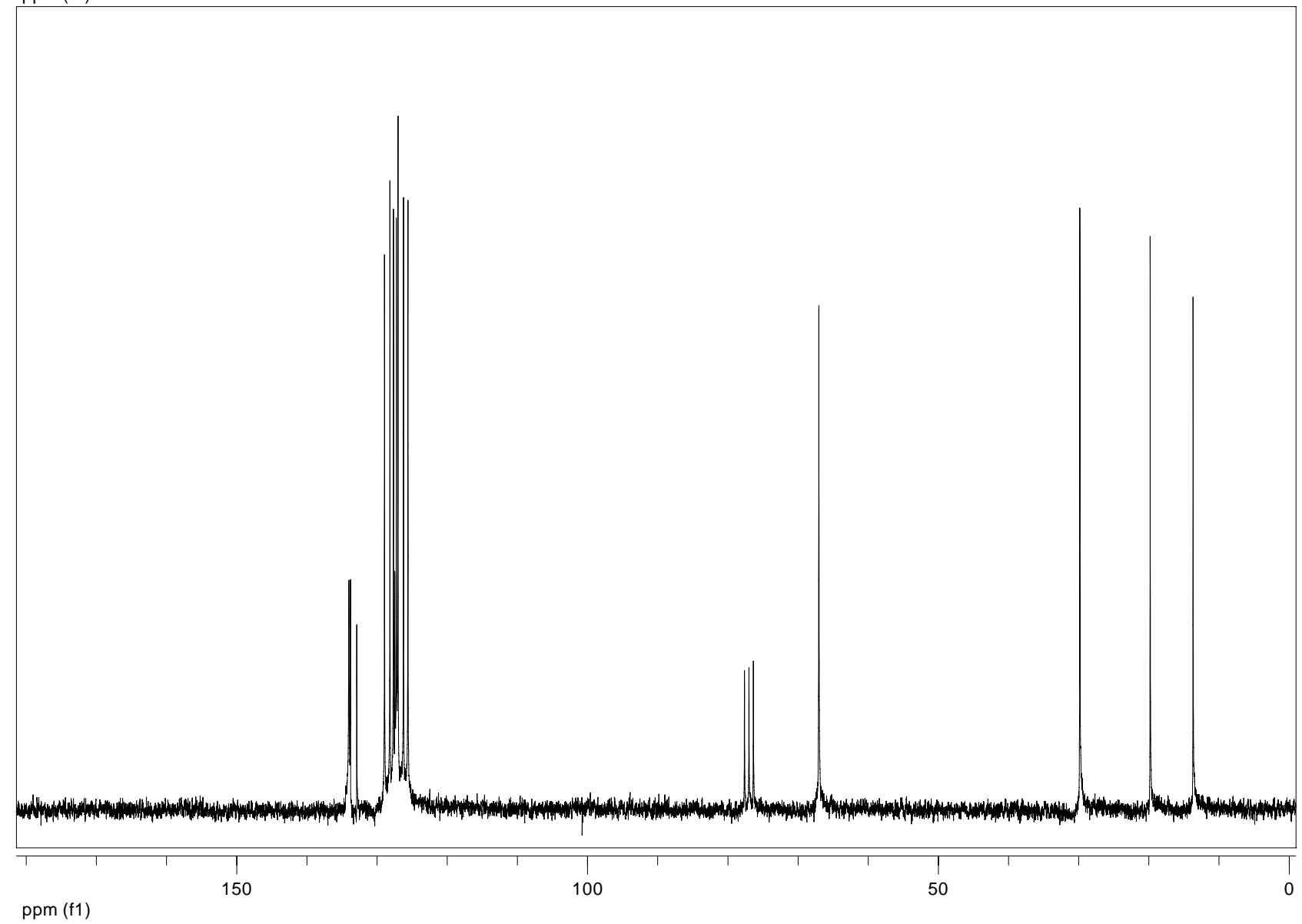




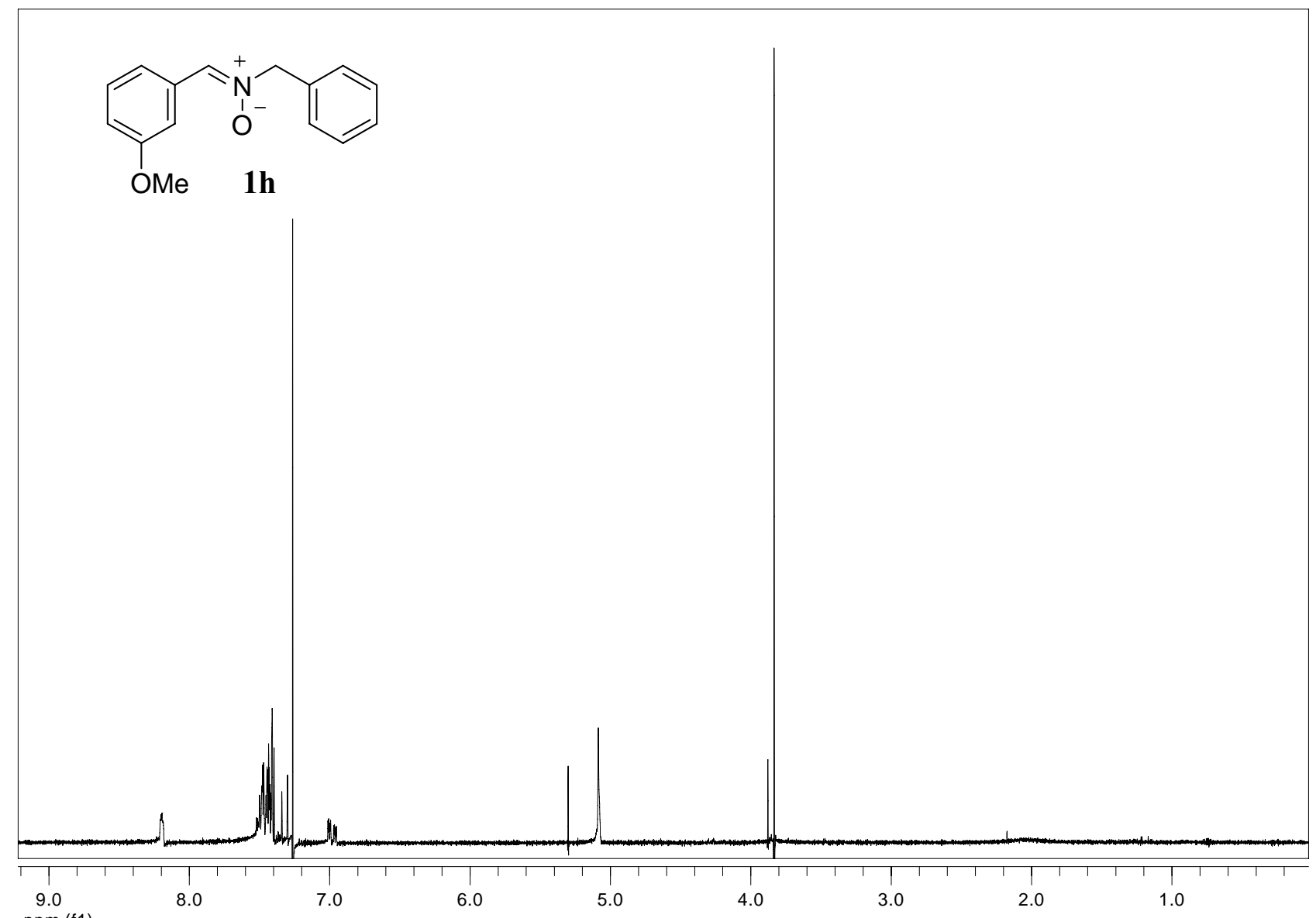

ppm (f1)

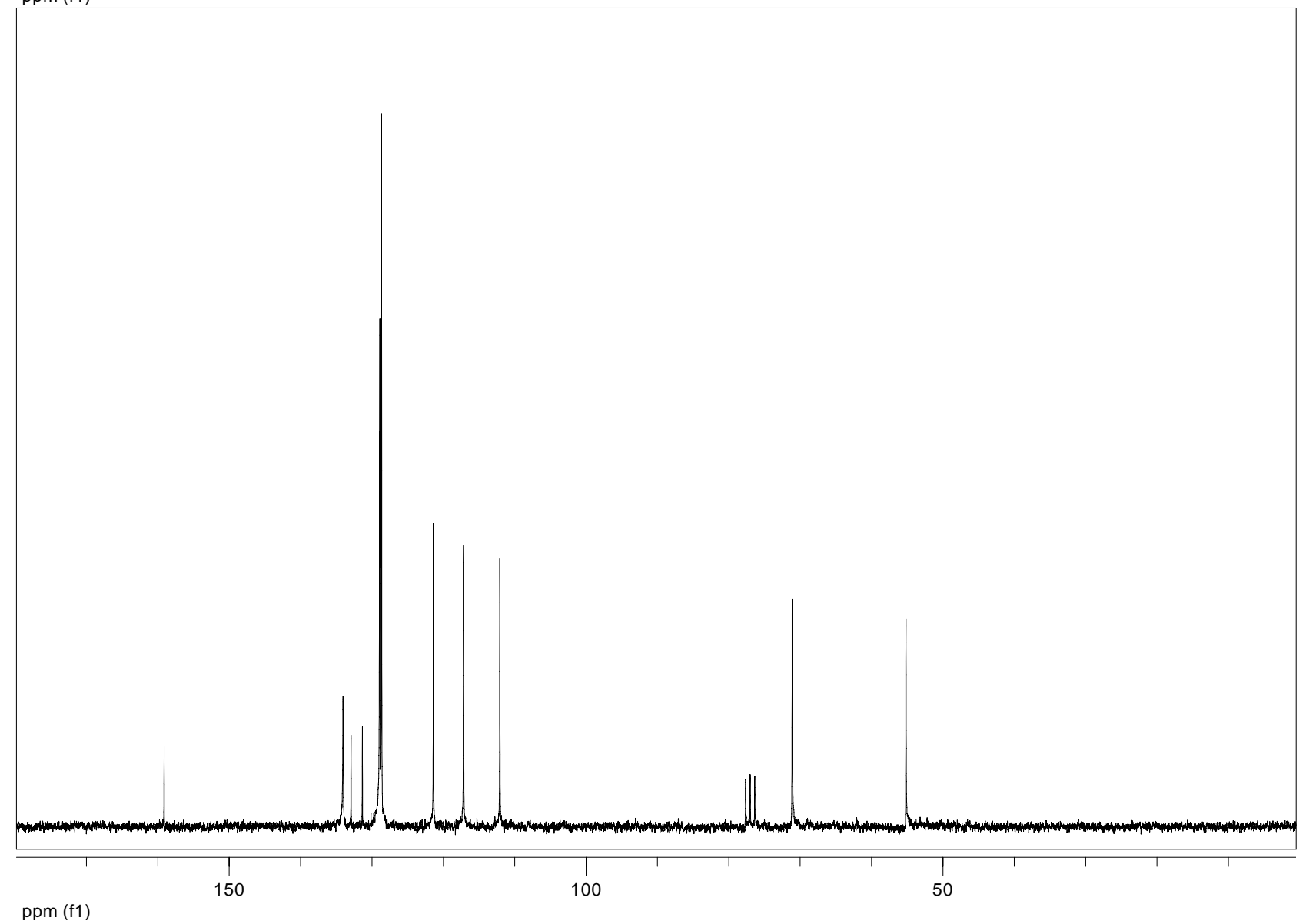




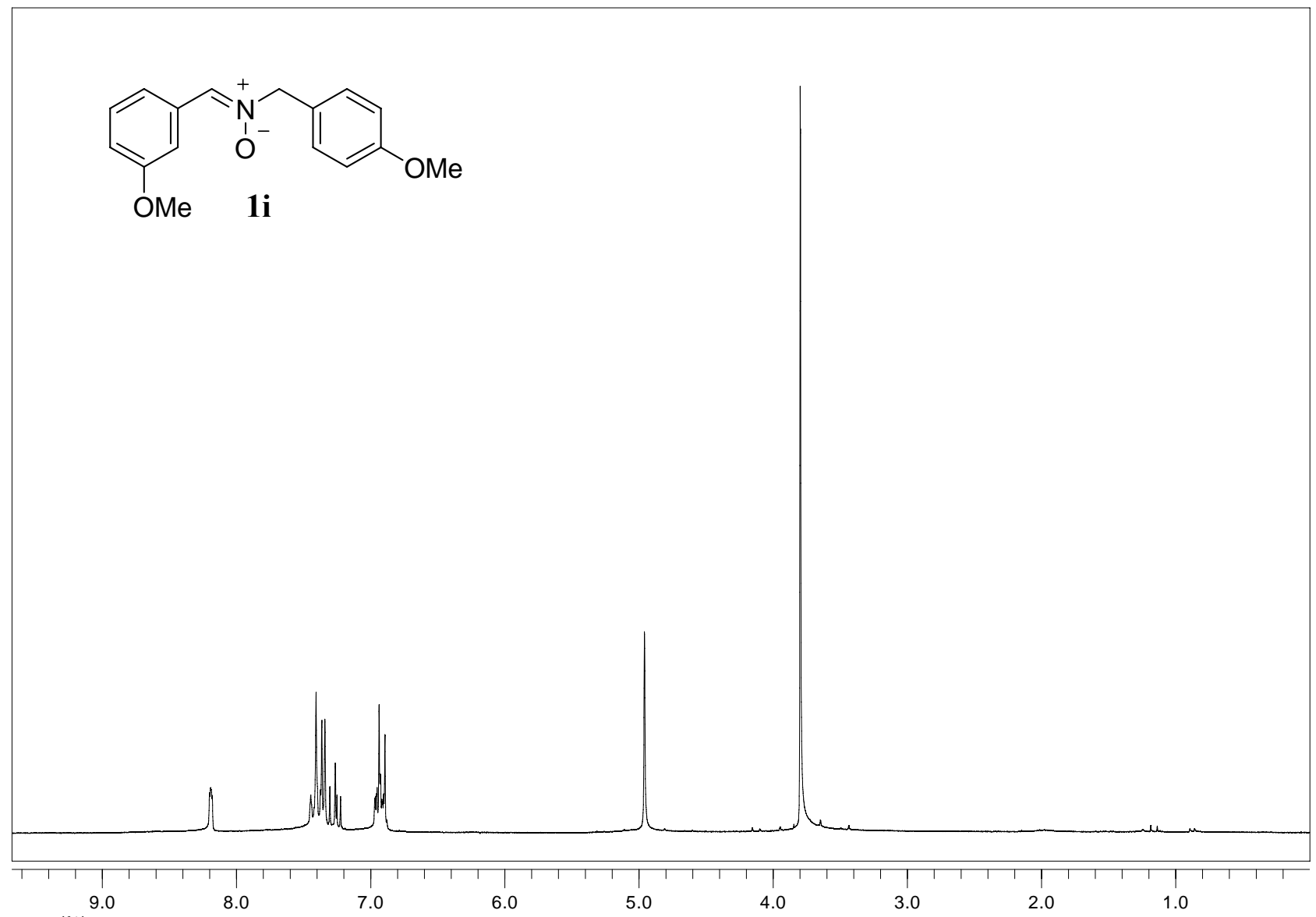

ppm (f1)

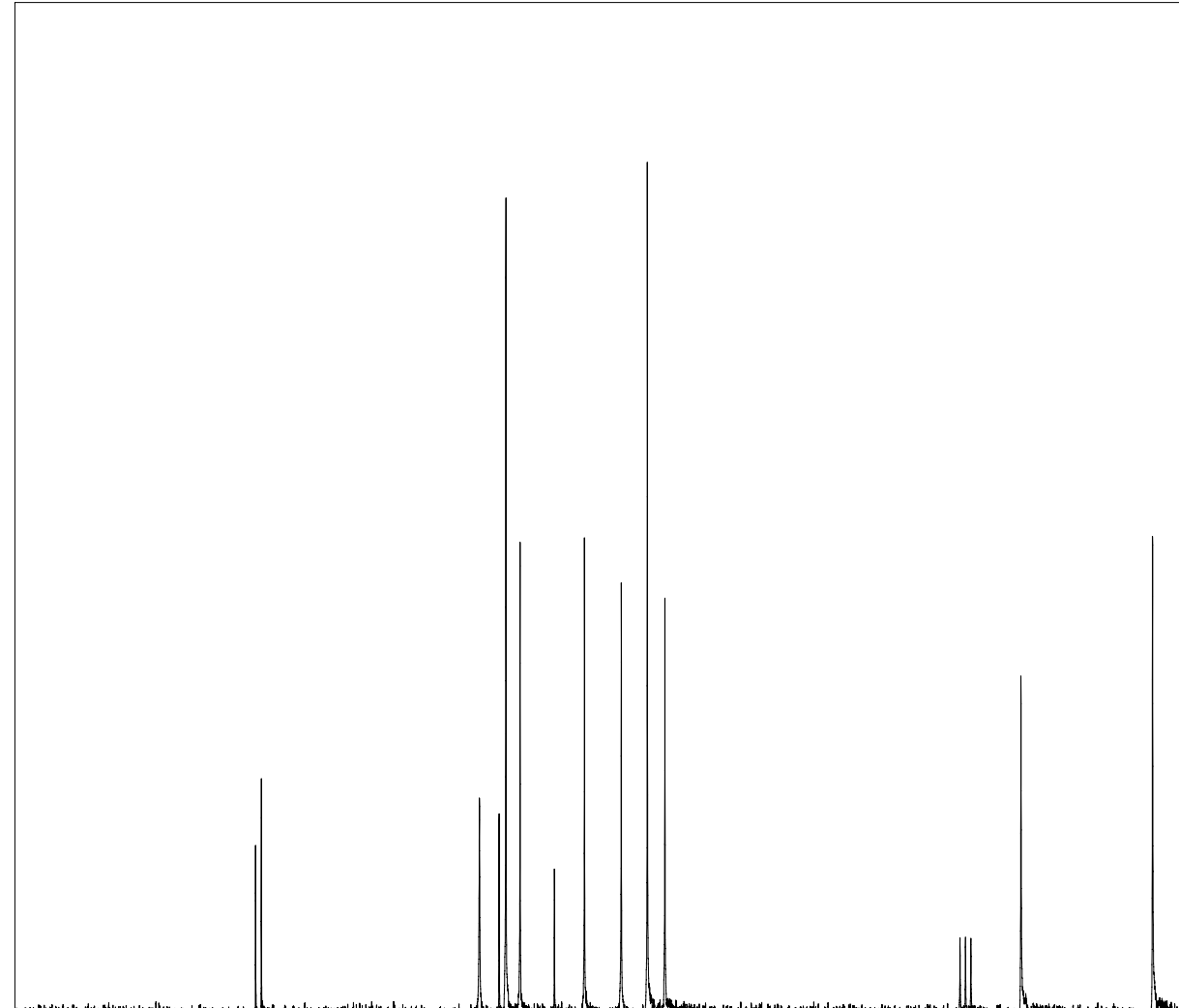

(1)

ppm (f1) 


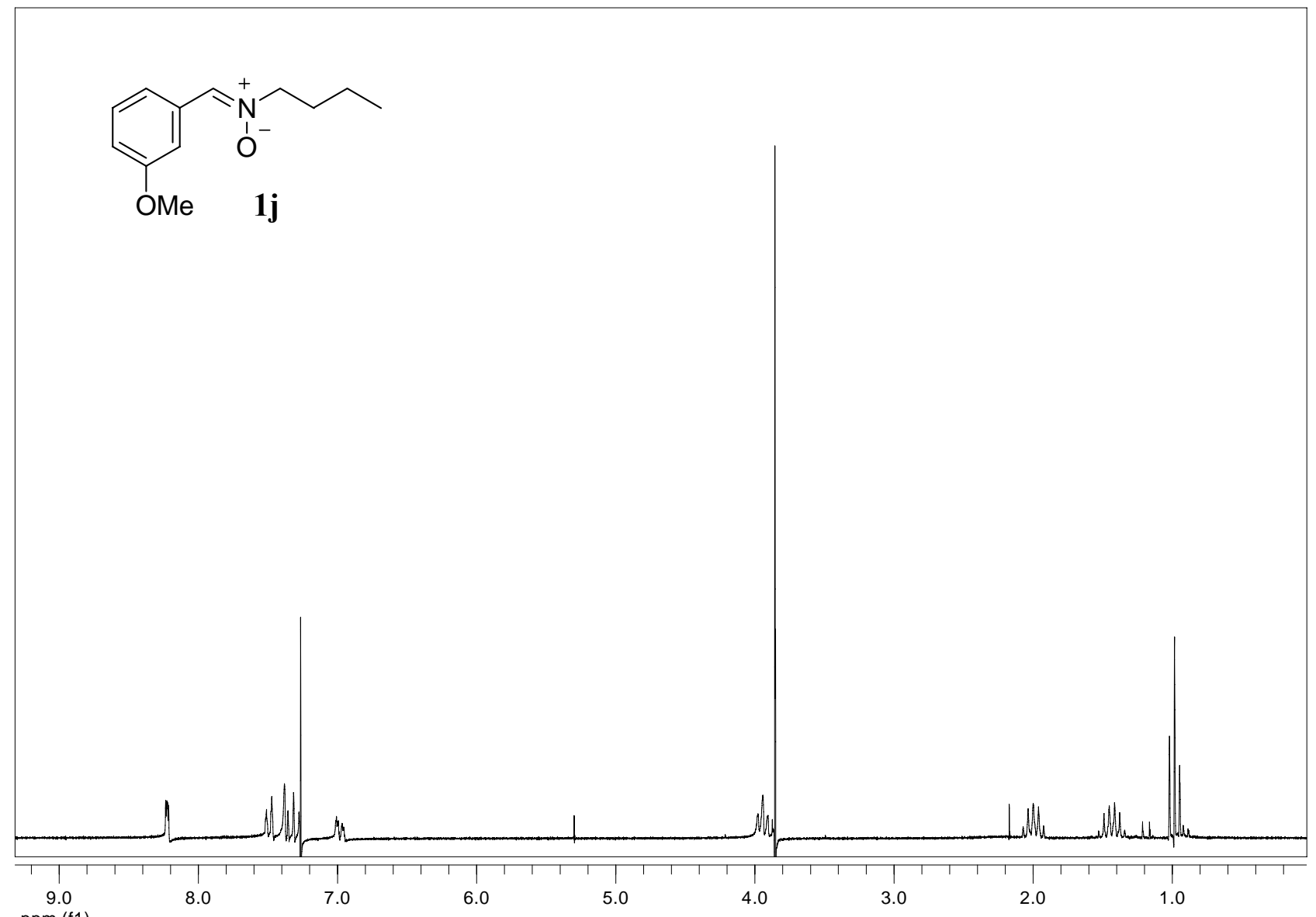

ppm (f1)

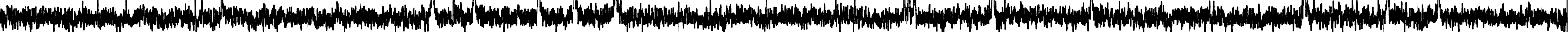

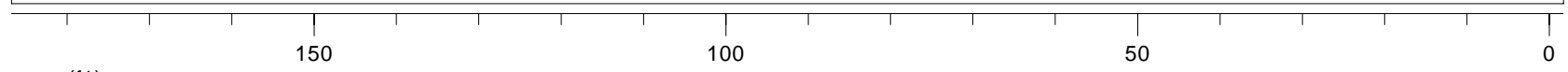

ppm (f1) 


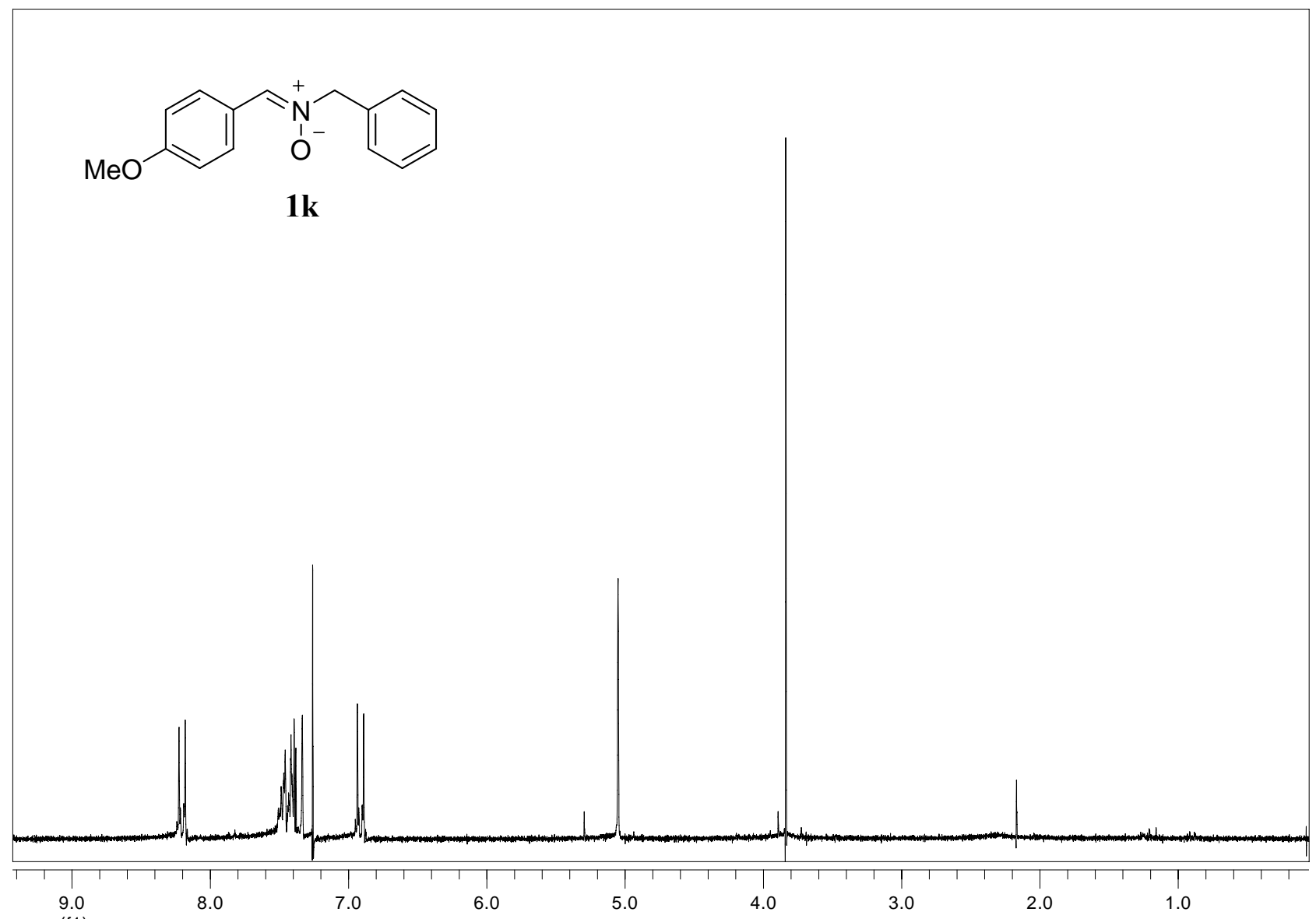

ppm (f1)

3.0

1.0

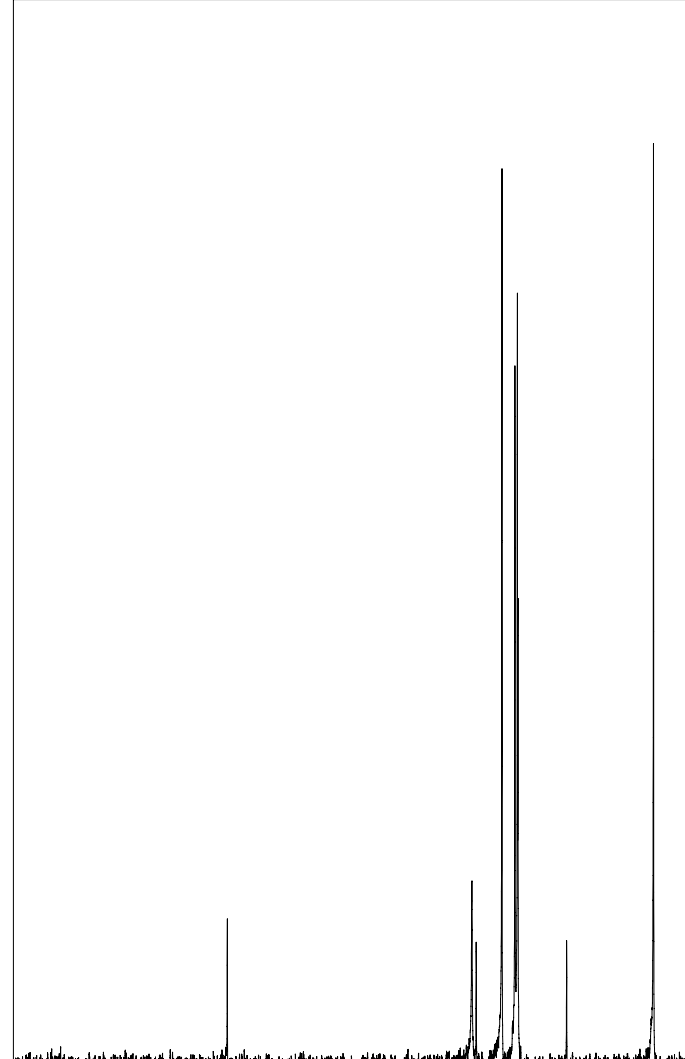

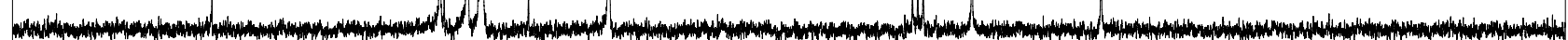<smiles>C1=CC=C1</smiles>

ppm (f1) 


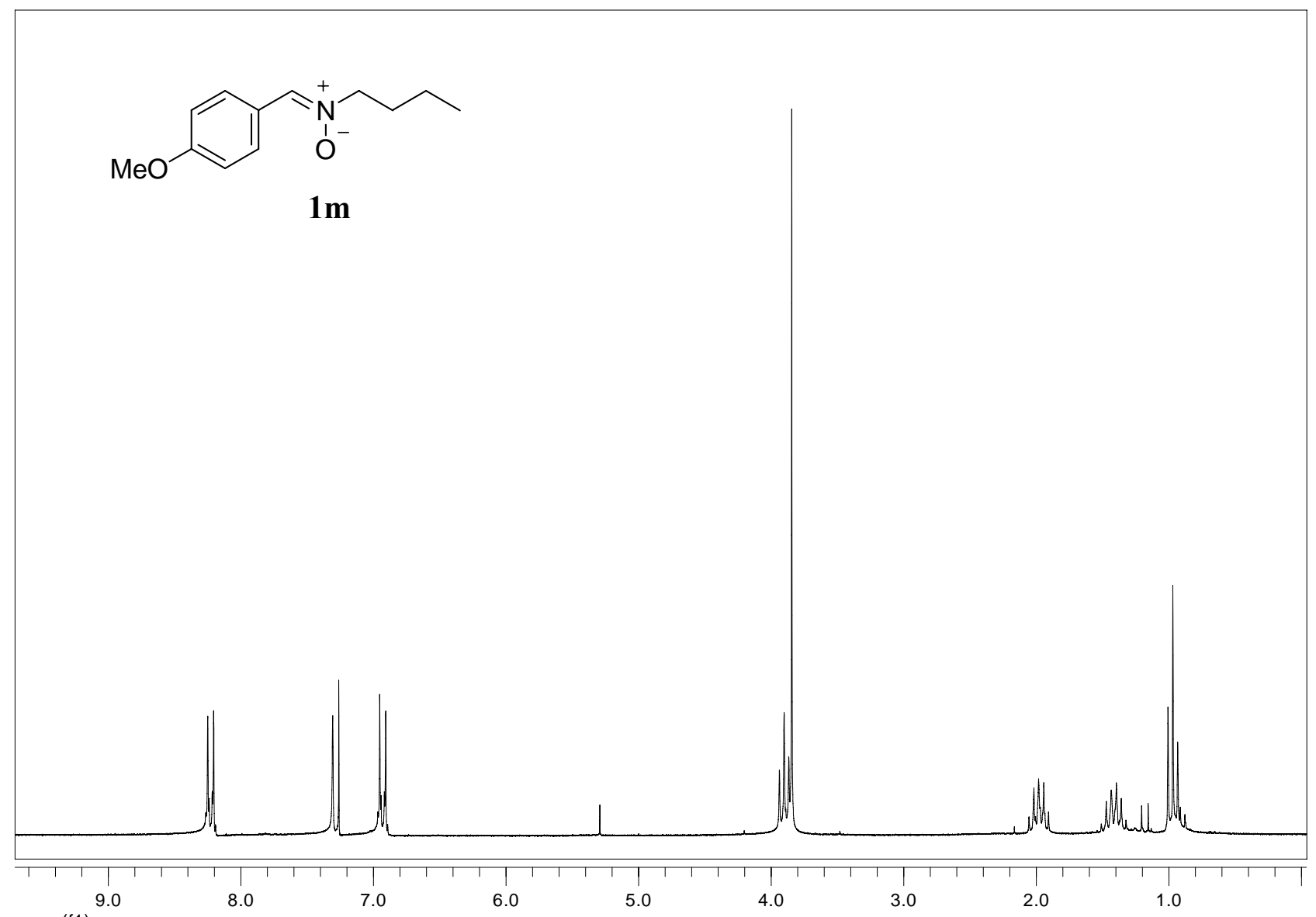

ppm (f1)

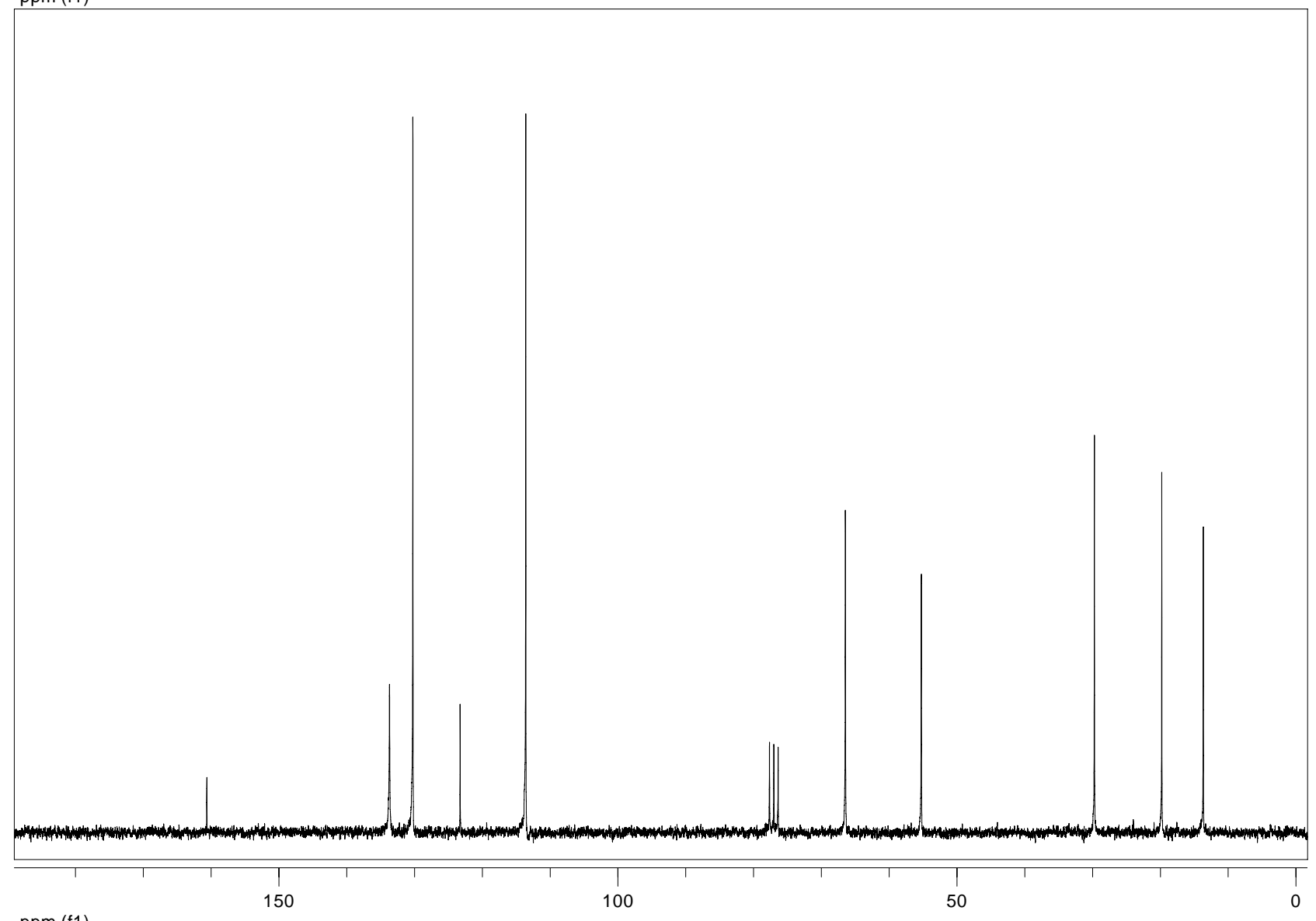

ppm (f1) 


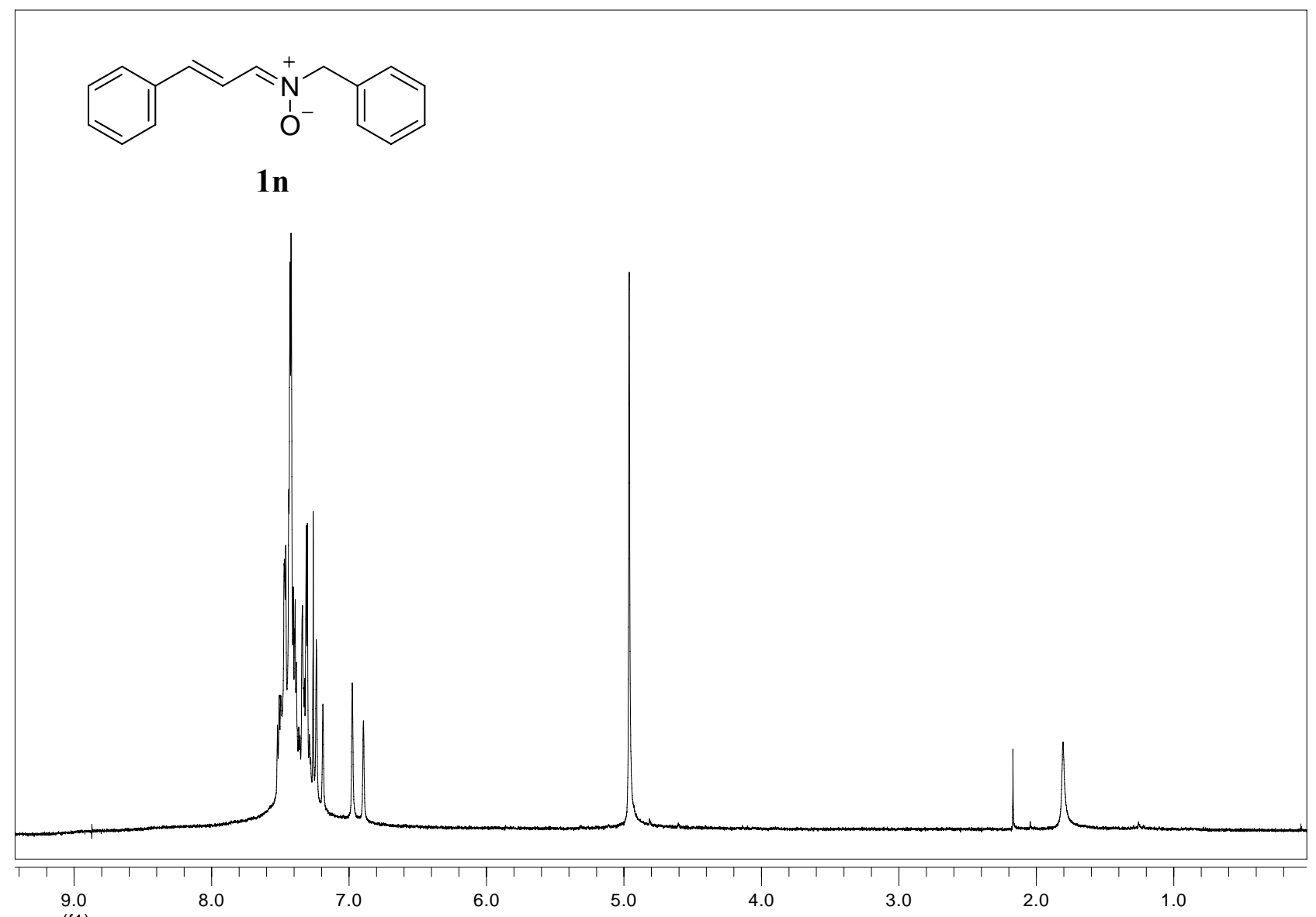
ppm (f1)

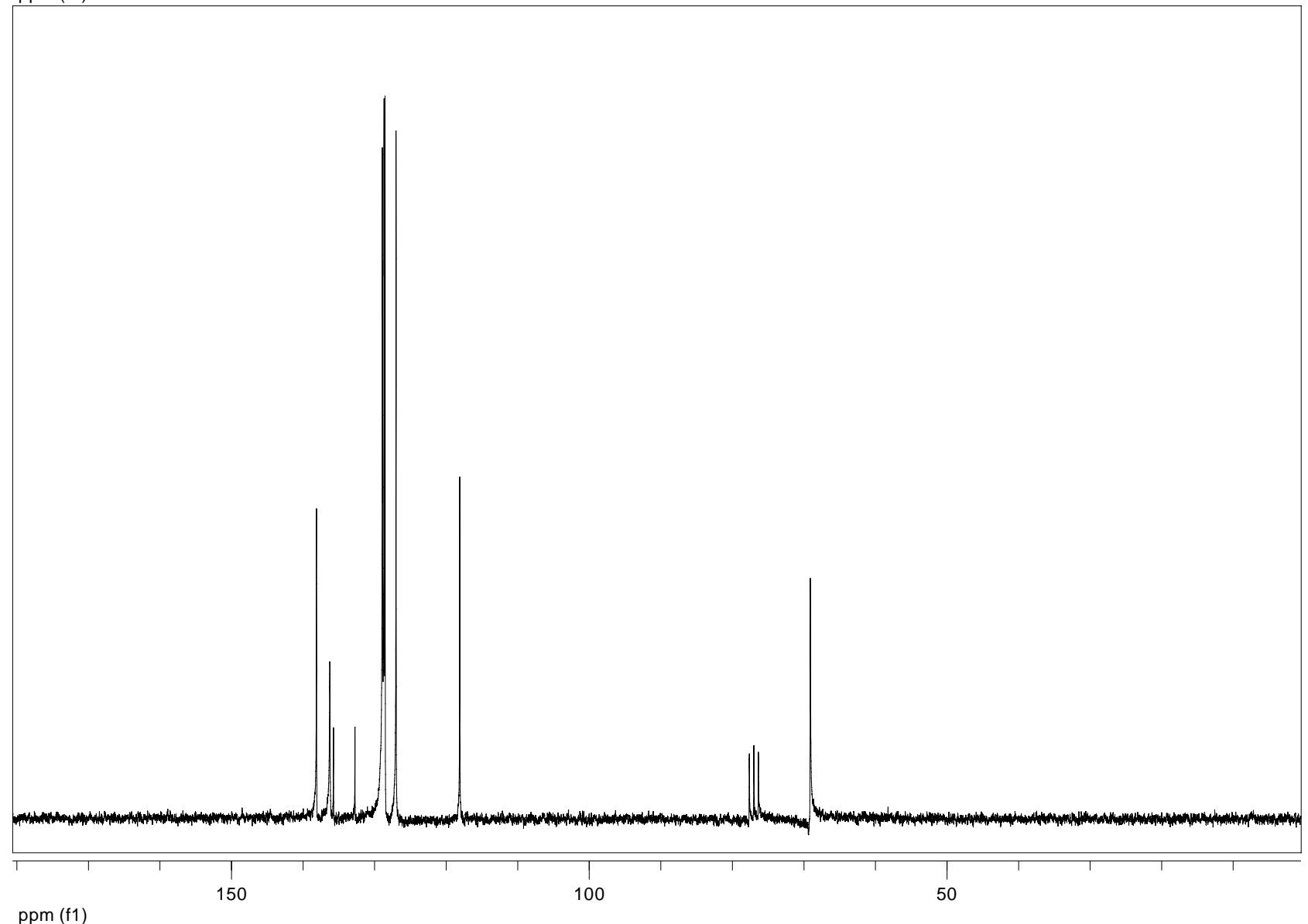




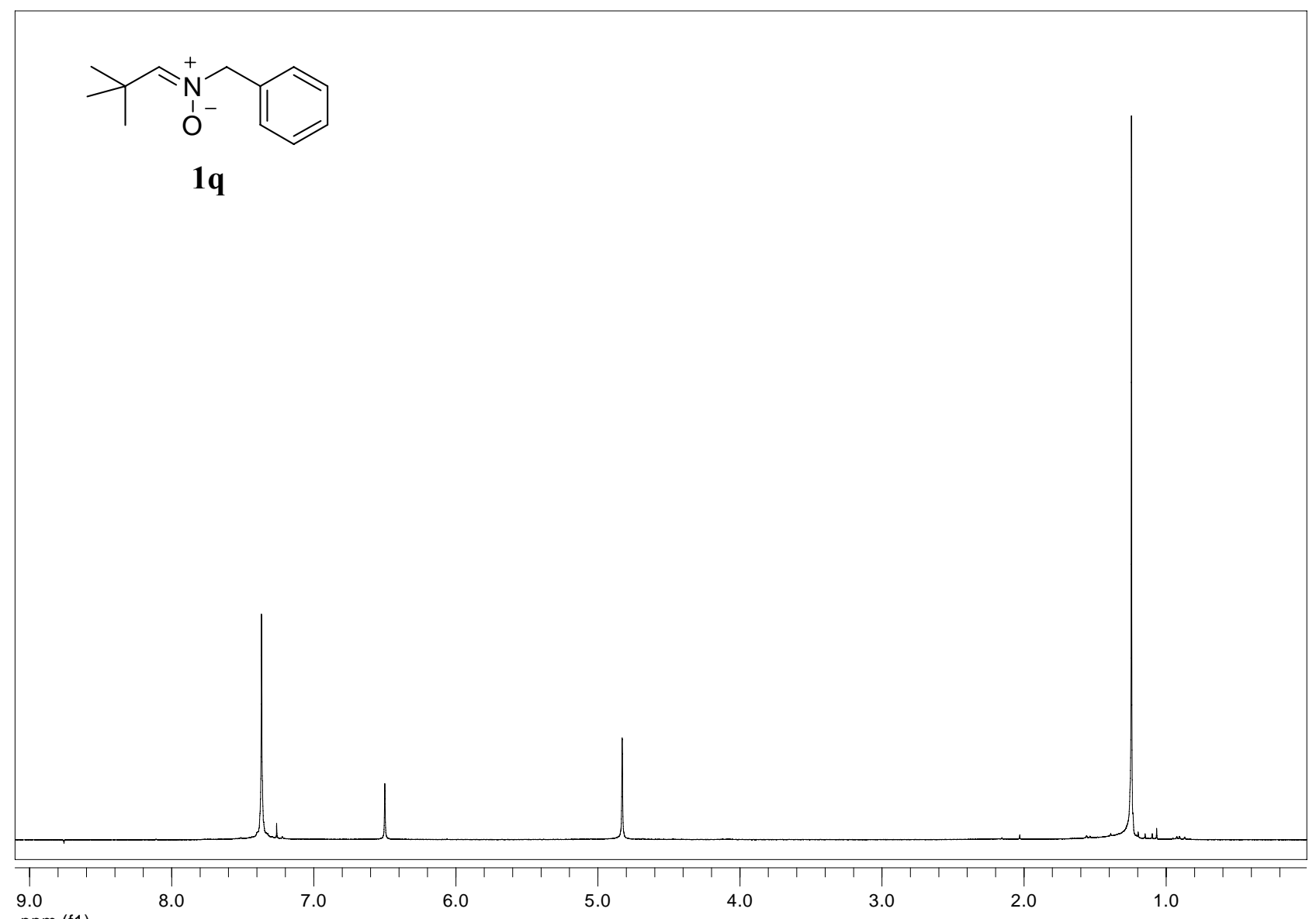

ppm (f1)

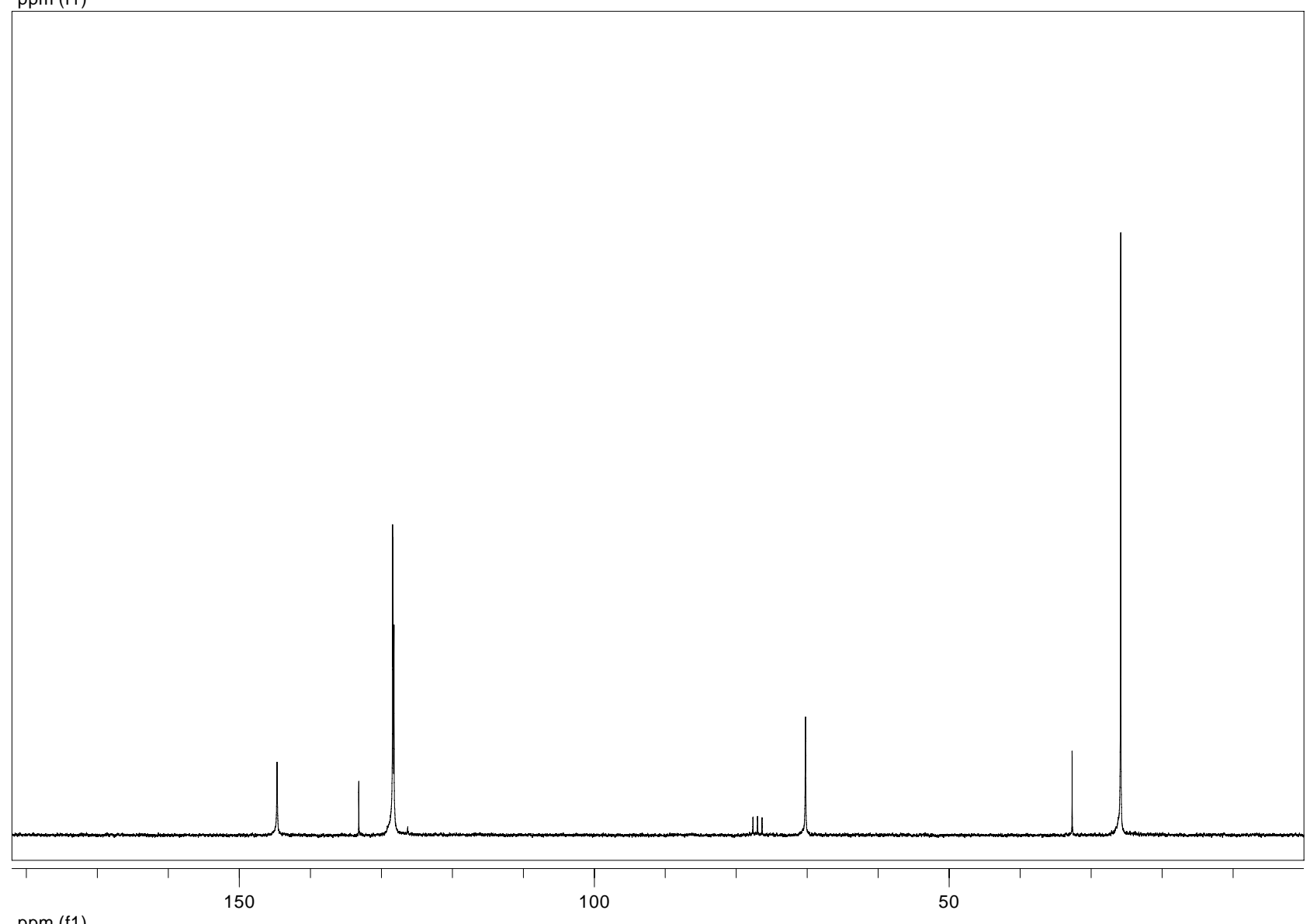

ppm (f1) 


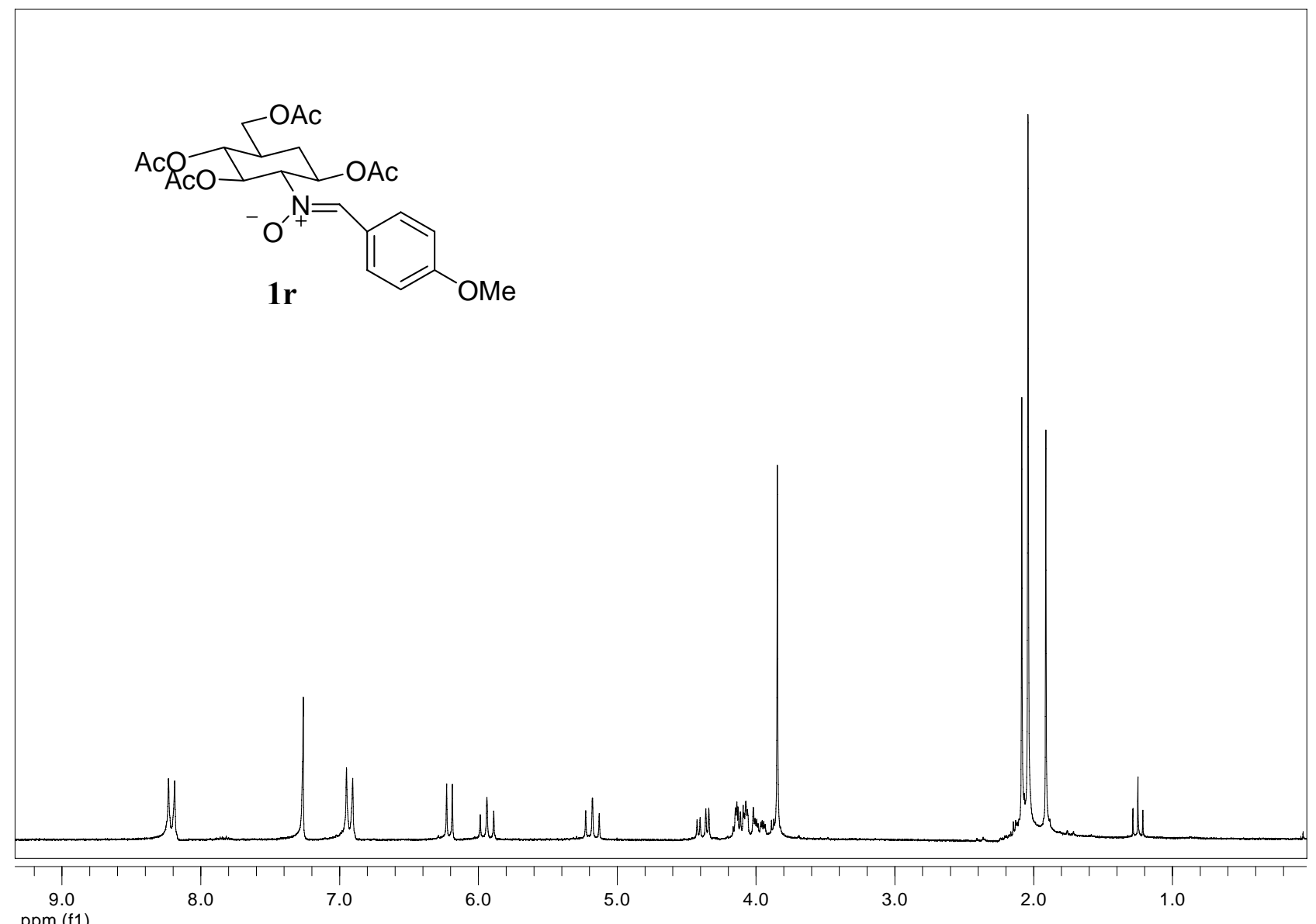

ppm (f1)

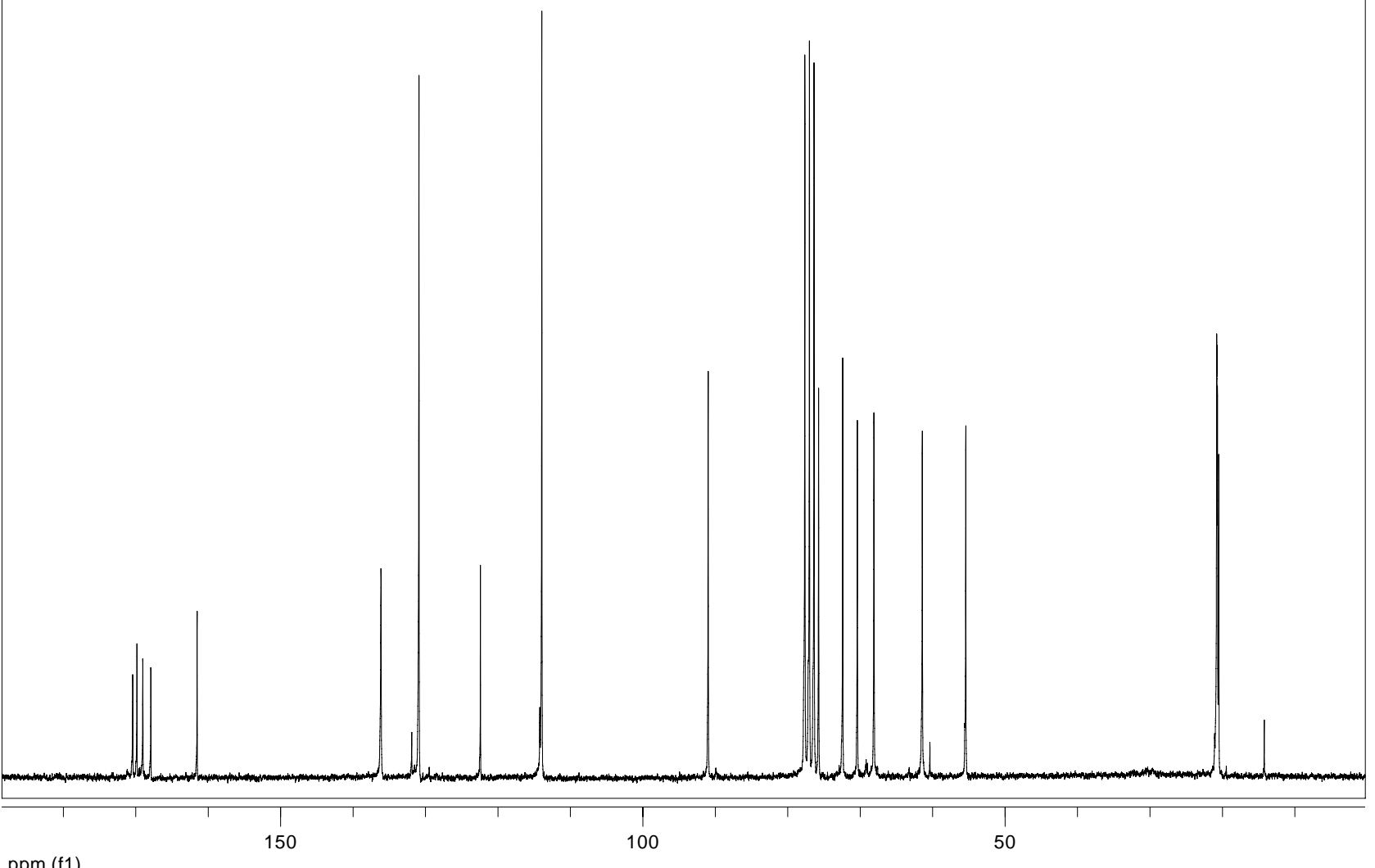

ppm (f1) 


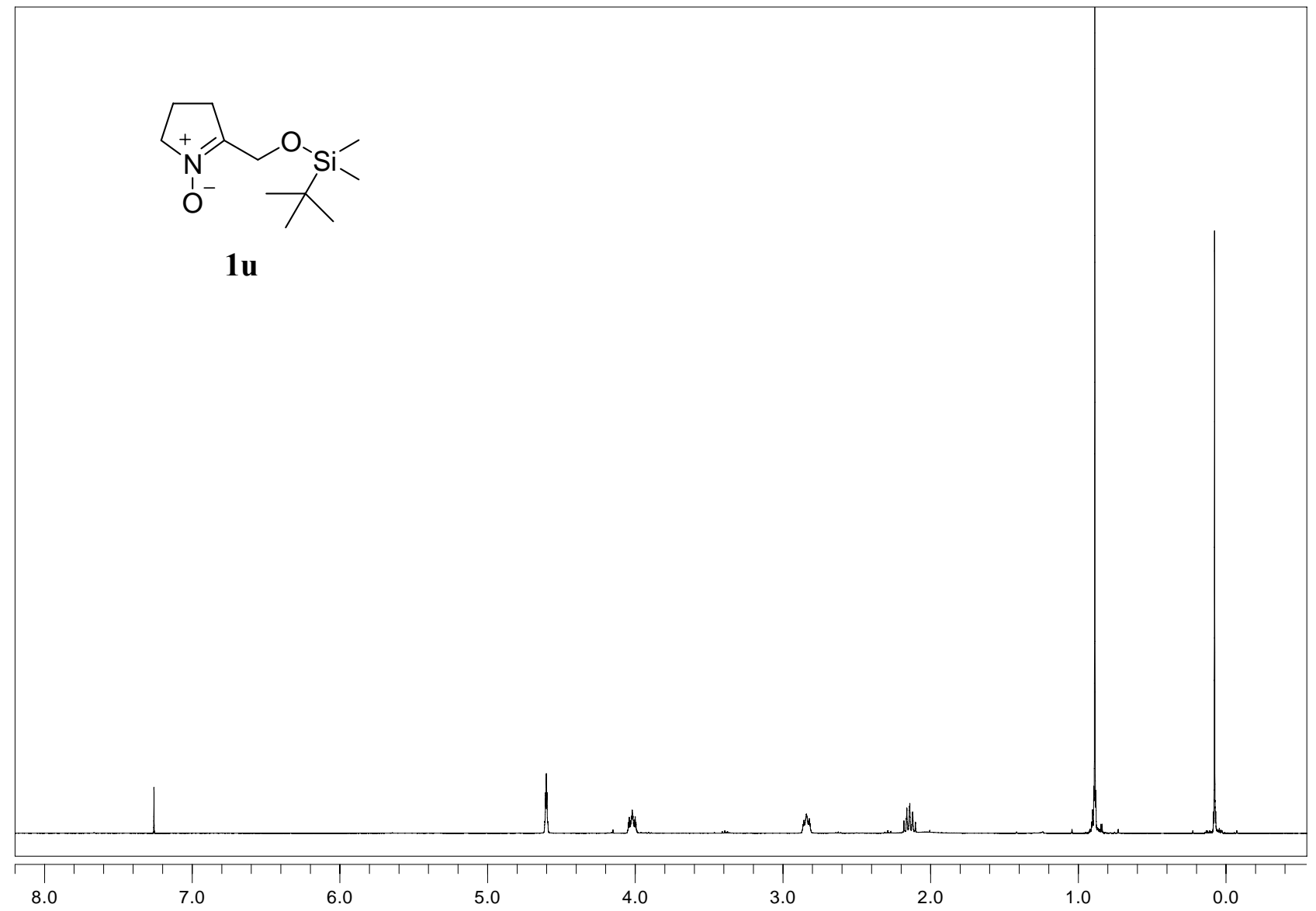

ppm (f1) 


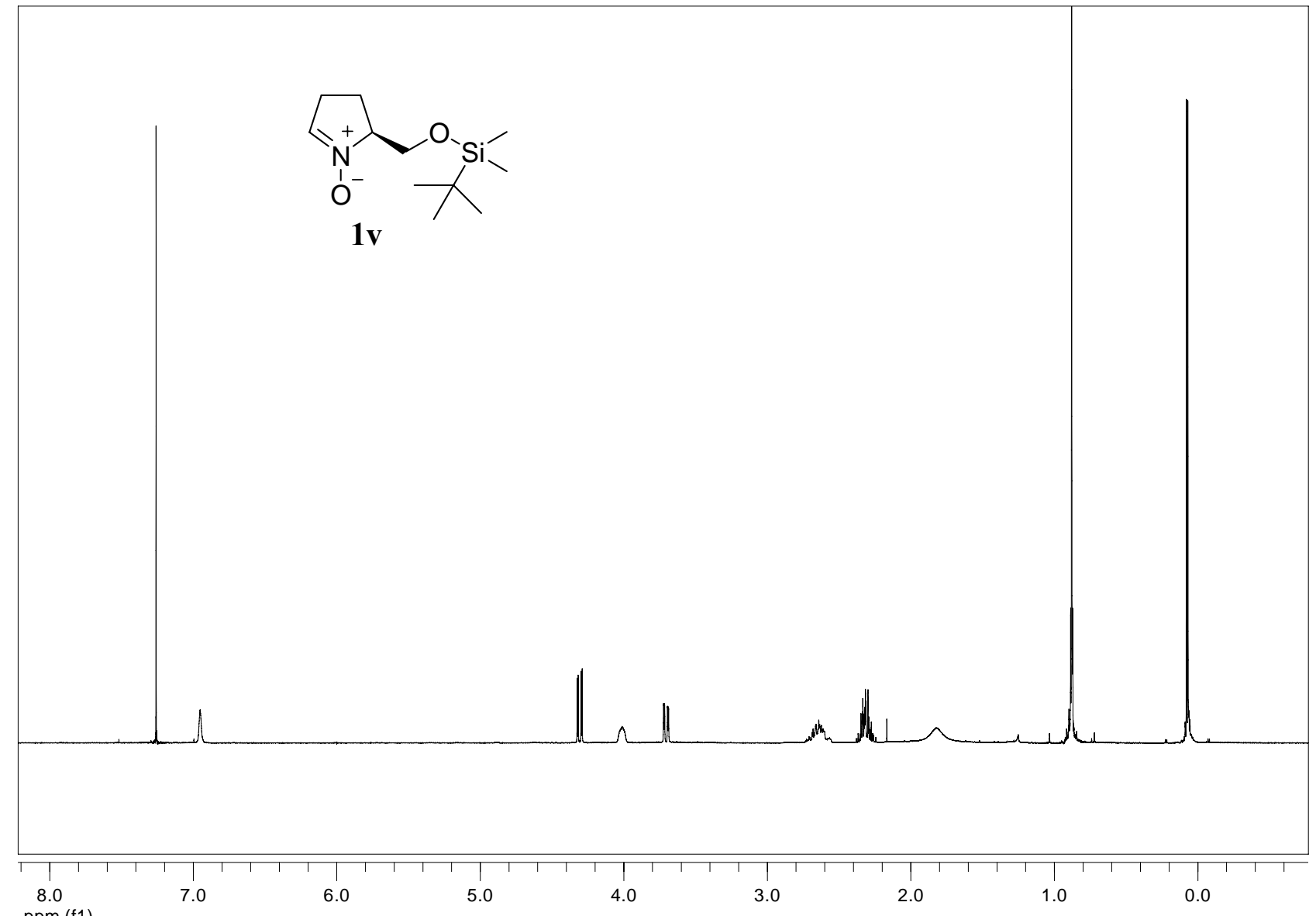

ppm (f1)

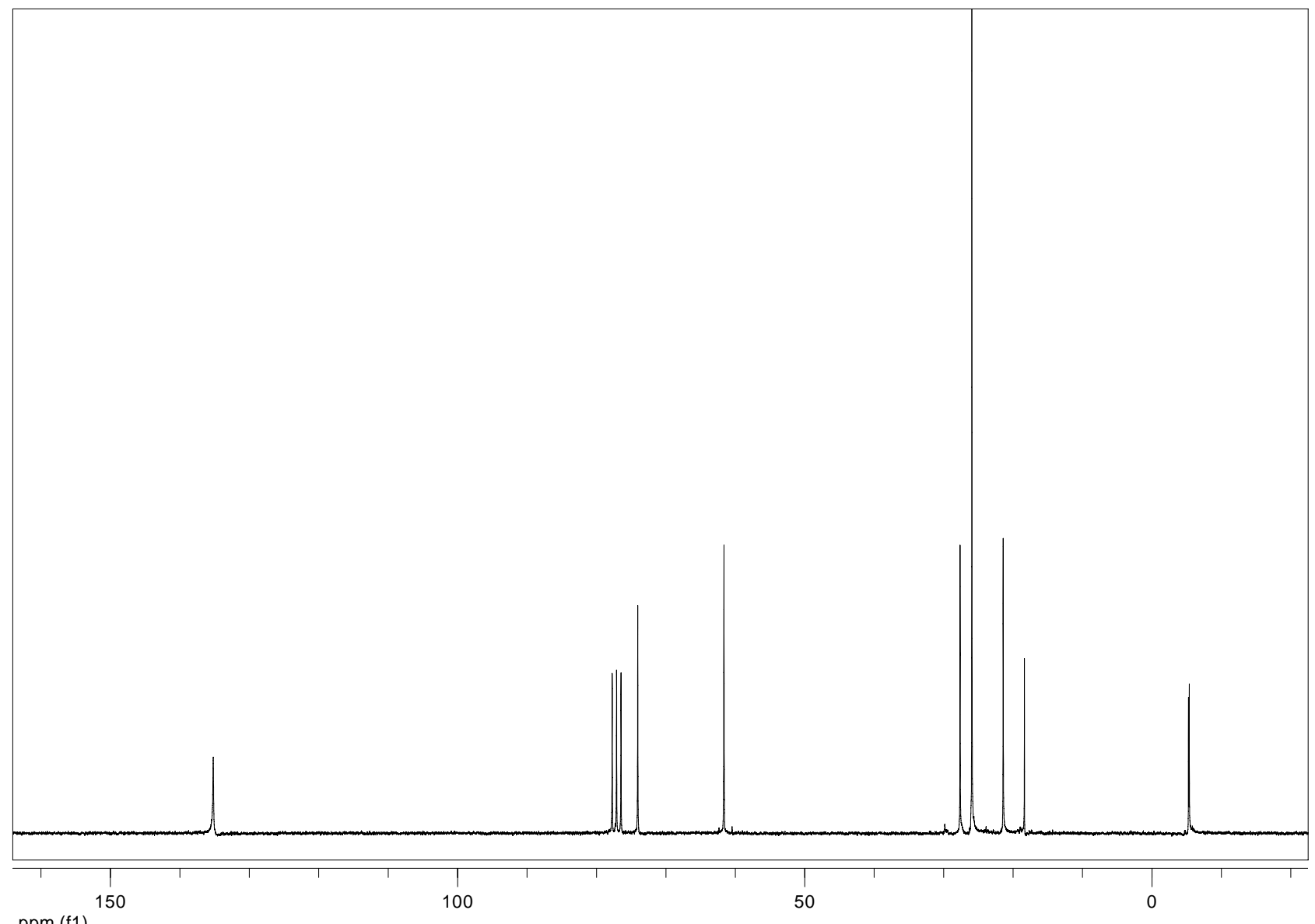

ppm (f1) 